Published in final edited form as:

J Control Release. 2015 June 10; 207: 18-30. doi:10.1016/j.jconrel.2015.03.033.

\title{
Exosomes as Drug Delivery Vehicles for Parkinson's Disease Therapy
}

\author{
Matthew J. Haney ${ }^{1,2}$, Natalia L. Klyachko ${ }^{1,2,3}$, Yuling Zhao ${ }^{1,2}$, Richa Gupta ${ }^{1,2}$, Evgeniya G. \\ Plotnikova $^{3}$, Zhijian He ${ }^{1,2}$, Tejash Patel ${ }^{2}$, Aleksandr Piroyan ${ }^{1,2}$, Marina Sokolsky ${ }^{1,2}$, \\ Alexander V. Kabanov ${ }^{1,2,3}$, and Elena V. Batrakova ${ }^{1,2, *}$ \\ ${ }^{1}$ Center for Nanotechnology in Drug Delivery, University of North Carolina at Chapel Hill, Chapel \\ Hill, North Carolina, USA \\ ${ }^{2}$ Eshelman School of Pharmacy, University of North Carolina at Chapel Hill, Chapel Hill, North \\ Carolina, USA \\ ${ }^{3}$ Deparment of Chemical Enzymology, Faculty of Chemistry, M.V. Lomonosov Moscow State \\ University, Moscow, Russia
}

\begin{abstract}
Exosomes are naturally occurring nanosized vesicles that have attracted considerable attention as drug delivery vehicles in the past few years. Exosomes are comprised of natural lipid bilayers with the abundance of adhesive proteins that readily interact with cellular membranes. We posit that exosomes secreted by monocytes and macrophages can provide an unprecedented opportunity to avoid entrapment in mononuclear phagocytes (as a part of the host immune system), and at the same time enhance delivery of incorporated drugs to target cells ultimately increasing drug therapeutic efficacy. In light of this, we developed a new exosomal-based delivery system for a potent antioxidant, catalase, to treat Parkinson's disease (PD). Catalase was loaded into exosomes ex vivo using different methods: the incubation at room temperature, permeabilization with saponin, freeze-thaw cycles, sonication, or extrusion. The size of the obtained catalase-loaded exosomes (exoCAT) was in the range of $100-200 \mathrm{~nm}$. A reformation of exosomes upon sonication and extrusion, or permeabilization with saponin resulted in high loading efficiency, sustained release, and catalase preservation against proteases degradation. Exosomes were readily taken up by neuronal cells in vitro. A considerable amount of exosomes was detected in PD mouse brain following intranasal administration. ExoCAT provided significant neuroprotective effects in in vitro and in vivo models of PD. Overall, exosome-based catalase formulations have a potential to be a versatile strategy to treat inflammatory and neurodegenerative disorders.
\end{abstract}

\footnotetext{
(C) 2015 Published by Elsevier B.V.

*Correspondence should be addressed to E.V.B., UNC Eshelman School of Pharmacy, University of North Carolina at Chapel Hill, Chapel Hill, NC 27599-7362, Phone: 919-537-3712, batrakov@email.unc.edu.

Publisher's Disclaimer: This is a PDF file of an unedited manuscript that has been accepted for publication. As a service to our customers we are providing this early version of the manuscript. The manuscript will undergo copyediting, typesetting, and review of the resulting proof before it is published in its final citable form. Please note that during the production process errors may be discovered which could affect the content, and all legal disclaimers that apply to the journal pertain.
} 


\section{Keywords}

blood-brain barrier; catalase; exosomes; neuroinflammation; oxidative stress; Parkinson's disease

\section{INTRODUCTION}

Parkinson's disease (PD) is one of the fastest growing neurological disorders in the developed world. The expected increase in lifespan of the population will further lead to a rise in age-related diseases, including neurodegenerative disorders. PD is known to be associated with brain inflammation, microglia activation and secretory neurotoxic activities, including reactive oxygen species (ROS) [1-4]. Samples from PD brains have shown reduced levels of redox enzymes, catalase and superoxide dismutase, and other antioxidants [5-7]. This may cause oxidative stress and neurodegeneration in PD patients. In this regard, catalase is one of the most potent in nature antioxidant that deactivates one million free radicals per second per molecule catalase in a cycle of catalytic reaction. As such, a successful brain delivery of catalase may be instrumental for PD therapy.

Regrettably, the blood brain barrier (BBB) severely limits transport of this large protein. In fact, $98 \%$ of all potent drugs that may improve therapy of various diseases of central nervious system (CNS) are not in clinic because of their inability to cross the BBB [8]. Different drug nanoformulations have been developed to overcome the BBB $[9,10]$. Unfortunately, the opsonization of drug-loaded nanoparticles in the bloodstream caused two main problems of drug nanoformulations: nanotoxicity and rapid drug clearance by mononuclear phagocyte system (MPS) [11]. To solve this problem, a polyethyleneglycol (PEG) corona was introduced to perpetuate a stealth effect. However, although the PEGylation decreased drug uptake by MPS, it concurrently reduced the interaction with target and barrier cells, thus, decreasing drugs biodistribution, particularly, in the brain [12-14]. In addition, the development of immune response to PEG corona significantly increased clearance of PEGylated drug nanocarriers [15-17]. For example, PEGylated liposomes were reported to lose their long-circulating property on the second week following systemic administration in mice [17]. This may become a real problem in case of chronic disease conditions, such as PD that requires a prolonged drug treatment. Furthermore, it was reported that $22 \%-25 \%$ healthy blood donors already have pre-existing PEG antibodies due to the everyday exposure to PEG in cosmetics, food, etc [18, 19]. In this respect, several reports indicated that exosomes may have an immune privileged status that can efficiently decrease drug clearance [20-22]. Thus, exosome-based nanocarriers may function as an "invisibility cloak" for incorporated therapeutic agents; they can diminish clearance by MPS, and at the same time increase drug transport to the brain.

Regarding their nature, exosomes are nanosized vesicles secreted by a variety of cells [23], in particular, cells of the immune system: dendritic cells [24], macrophages [25], B cells [26], and T cells [27]. Exosomes were initially thought to be a mechanism for removing unneeded proteins. Recent studies revealed, they are actually specialized in long-distance intercellular communications facilitating transfer of proteins [28], functional mRNAs and microRNAs for subsequent protein expression in target cells [29, 30]. To shuttle their cargo, 
exosomes can attach by a range of surface adhesion proteins and specific vector ligands (tetraspanins, integrins, $\mathrm{CD} 11 \mathrm{~b}$ and $\mathrm{CD} 18$ receptors), and deliver their payload to target cells $[24,31]$. Noteworthy, exosomes possess an intrinsic ability to cross biological barriers.

Thus, tumor-derived exosomes and microvesicles originated in the brain of glioma-bearing mice, and human glioblastoma patients were detected in the blood circulation indicating their ability to cross the BBB [32].

Exosomes were exploited as drug delivery vehicles in several investigations [33-37]. In particular, exosomes loaded with an anti-inflammatory low molecular drug, curcumin, were shown to protect mice from lipopolysaccharides-induced brain inflammation [36]. Moreover, exosomes were harnessed for systemic delivery of exogenous siRNA across the biological barriers [20,33, 37-39]. The incorporation of the therapeutic agents into exosomes increased the circulation time, preserved drug therapeutic activity, and improved the brain delivery. The similar methodology was applied to polymer nanoparticles that were covered with erythrocytes membranes [40]. Sheltering of nanoparticles with cellular membranes/lipids produced a potent stealth effect increasing their time circulation in the blood. Furthermore, cellular membranes of monocytes and macrophages were utilized to manufacture nanovesicles by their serial extrusion through filters with diminishing pore sizes [41]. According to this report, cell-derived nanovesicles loaded with anticancer agent, doxorubicin (Dox) significantly reduced tumor growth in mice without adverse effects that were reported for equipotent free Dox. Noteworthy, the effects of Dox-loaded exosomes were superior than the commercially available Dox-loaded liposomes, Doxil; the liposomal formulation was inefficient in reducing tumor growth in this model [41]. Noteworthy, exosomes can exert unique biological activity reflective of their origin. According to a recent study, exosomes released from mesenchymal stem cells produced significant cardioprotective paracrine effects against myocardial ischemia/reperfusion injury even without any drug loaded [42]. Overall, these reports indicate that exosomes may function as exceptional vehicles for the delivery of therapeutic agents.

We demonstrated earlier that macrophages preloaded with nanoformulated catalase, or transfected with catalase-encoded plasmid DNA can release exosomes with incorporated catalase, and facilitate drug transfer to target cells of neurovascular unit: neurons, astrocytes, and brain microvessel endothelial cells [43-45]. This process resulted in profound therapeutic effects in mouse models of PD [44, 46]. We report here the development of a new exosomal-based formulation of catalase (exoCAT) that was obtained by drug loading into naïve exosomes ex vivo. Different approaches for drug incorporation were validated: incubation at room temperature (RT) with or without saponin permeabilization, freeze-thaw cycles, sonication, or extrusion of exosomes in the presence of catalase. The obtained exoCAT formulations were evaluated for catalase loading efficiency, release and antioxidant activity. Selected optimal exoCAT efficiently decreased oxidative stress and increased neuronal survival in in vitro and in vivo models of PD. We hypothesized that the encapsulation of catalase into exosomes may preserve catalase enzymatic activity, prolong blood circulation time, and reduce immunogenicity, thereby improve drug therapeutic efficacy. Importantly, this technology may be more generally applicable to the treatment of many CNS diseases, in particular neurodegenerative disorders [47-49] such as PD, 
Alzheimer's disease, infectious diseases (meningitis, encephalitis, and HIV-associated neurocognitive disorders [50, 51]), stroke [52, 53], and lysosomal storage diseases [54, 55].

\section{MATERIALS AND METHODS}

\subsection{Reagents}

Catalase from bovine liver was purchased from Calbiochem (San Diego, CA). A lipophilic fluorescent dyes, 1,1'-dioctadecyl-3,3,3',3'-tetramethylindo-carbocyanine perchlorate (DIL), and 2-(5-(1,3-dihydro-3,3-dimethyl-1-octadecyl-2H-indol-2-ylidene)-1,3-pentadienyl)-3,3dimethyl-1-octadecyl-perchlorate (DID) were purchased from Invitrogen (Carlsbad, CA, USA). 6-hydroxydopamine (6-OHDA), lipopolysaccharides (LPS), rhodamine isothiocyanate (RITC), and Triton X-100 were obtained from Sigma-Aldrich (St. Louis, MO, USA). Interferon gamma (INT- $\gamma$ ) was purchased from Peprotech Inc. (RockyHill, NJ, USA).

\subsection{Cells}

A mouse macrophage cell line (Raw 264.7) was purchased from American Type Culture Collection (ATCC, Manassas, VA, USA, cat \# TIB-71), and cultured in Dulbecco's Modified Eagle's Media (DMEM) (Invitrogen) supplemented with 10\% (v/v) heatinactivated fetal bovine serum (FBS). Neuronal PC12 rat adrenal pheochromocytoma cell line was obtained from ATCC, and cultured in Dulbecco's modified Eagle medium (Hyclone, South Logan, UT, USA) supplemented with $10 \%$ FBS, and $1 \%(\mathrm{v} / \mathrm{v})$ of both penicillin and streptomycin. The cells were grown in an incubator with optimal culture conditions of $37{ }^{\circ} \mathrm{C}$ and $5 \% \mathrm{CO}_{2}$, and the medium was routinely replaced every $2-3$ days.

Bone marrow derived macrophages (BMM) were obtained by differentiation of bone marrow stem cells extracted from murine femurs (C57BL/6, female mice) as described in [56]. The cells were cultured for 10 days in media supplemented with $1000 \mathrm{U} / \mathrm{mL}$ macrophage colony-stimulating factor (MCSF). The purity of monocyte culture was determined by flow cytometry using FACS Calibur (BD Biosciences, San Jose, CA, USA). Mouse primary cultured cortical neurons and dopaminergic (DA) neurons from substantia nigra pars compacta ( $S N p c$ ) were isolated from mouse pups cortex and midbrain as described [57].

\subsection{Isolation of exosomes}

Concomitant media from Raw 264.7 macrophages grown on $75 \mathrm{~T}$ flasks $\left(20 \times 10^{6}\right.$ cells/ flask) was collected, and exosomes were isolated using gradient centrifugation [24]. In brief, the culture supernatants were cleared of cell debris and large vesicles by sequential centrifugation at $300 \mathrm{~g}$ for $10 \mathrm{~min}, 1000 \mathrm{~g}$ for $20 \mathrm{~min}$, and 10,000g for $30 \mathrm{~min}$, followed by filtration using $0.2 \mu \mathrm{m}$ syringe filters. Then, the cleared sample was spun at $100,000 \mathrm{~g}$ for one hour to pellet the exosomes, and supernatant was collected. The collected exosomes $\left(10^{11}-10^{12}\right.$ exosomes/flask) were washed twice with phosphate buffer solution (PBS). To avoid contamination by the FBS-derived exosomes, FBS was spun at 100,000g for 2 hours to remove exosomes before the experiment. The recovery of exosomes was estimated by measuring the protein concentration using the Bradford assay and by Nanoparticle Tracking 
Analysis (NTA). The obtained exosomal fraction was re-suspended in PBS (500 $\mu \mathrm{l}, 1$ $\mathrm{mg} / \mathrm{mL}$ total protein), and characterized for size and polydispersity.

\subsection{Loading of Exosomes}

Four approaches for catalase incorporation into exosomes were evaluated: the incubation at RT with or without saponin (Method I), freeze-thaw cycles (Method II), sonication (Method III), and extrusion (Method VI). For Method I, naive exosomes released from Raw 264.7 macrophages were diluted in PBS to a concentration $0.15 \mathrm{mg} / \mathrm{mL}$ of total protein, then catalase solution in PBS $(0.5 \mathrm{mg} / \mathrm{mL})$ was added to $250 \mu \mathrm{l}$ of exosomes to the final concentration $0.1 \mathrm{mg} / \mathrm{mL}$ total protein, and incubated at RT for 18 hours. In case of a saponin treatment, a mixture of catalase and exosomes was supplemented with $0.2 \%$ saponin and placed on shaker for $20 \mathrm{~min}$ at RT. For Method II, the catalase solution was added to exosomes as described above, incubated for $30 \mathrm{~min}$, then rapidly freezed at $-80^{\circ} \mathrm{C}$, and thawed at RT. The freeze-thaw cycle was repeated three times. For Method III, the catalase mixture with exosomes was sonicated $(500 \mathrm{v}, 2 \mathrm{kHz}, 20 \%$ power, 6 cycles by $4 \mathrm{sec}$ pulse $/ 2$ sec pause), cooled down on ice for $2 \mathrm{~min}$, and then sonicated again using Qsonica Sonicator Q700 (Fisher Scientific, Hampton, NH, USA). For Method IV, catalase mixture with exosomes was extruded (x10 times) through Avanti Lipids extruder (Avanti Polar lipids Inc., Alabaster, AL, USA) with $200 \mathrm{~nm}$-pores diameter. Loaded with catalase exosomes were purified from free catalase by gel-filtration chromatography with Sepharose 6 BCL (Sigma-Aldrich).

\subsection{Characterization of different exoCAT formulations by (Dynamic Light Scattering) DLS, (Atomic Force Microscopy) AFM, Nanoparticle Tracking Analysis (NTA), and Hyperspectral microscopy}

The effective hydrodynamic diameter of empty exosomes, or exosomes loaded with catalase was measured by DLS using the ZetaPlus' Zeta Potential Analyzer (Brookhaven Instruments, Santa Barbara, CA, USA) equipped with a $35 \mathrm{~mW}$ solid state laser (658 $\mathrm{nm}$ laser) as described in $[58,59]$. The size, distribution, and number of particles for various exosomal formulations were also examined by NTA. For this purpose, exoCAT formulations were prepared at concentration $0.01 \mathrm{mg} / \mathrm{mL}$, and evaluated using NanoSight 500, Version 2.2 (Wiltshire, United Kingdom). The morphology of exoCAT aggregates was investigated by AFM. Different exoCAT formulations were prepared in $50 \mathrm{mM}$ phosphate buffer, pH 7.4 at total protein $10 \mu \mathrm{g} / \mathrm{mL}$. A drop of the sample was placed on a glass slide, and dried under an argon flow. The AFM imaging was operated as described earlier [60].

ExoCAT formulations were further characterized by Hyperspectral microscopy (CytoViva Inc., Auburn, AL, USA). The hyperspectral images and the corresponding hyperspectral data were captured using an Olympus BX43 research grade optical microscope equipped with the patented CytoViva advanced dark field illumination system and diffraction grating hyperspectral imaging system (CytoViva Inc.). A 100x oil iris .6-1.30 NA objective was utilized. For the mean spectral comparison, data was captured from multiple pixels within multiple exosome particle areas of each sample. The mean spectrum from each sample was calculated using the CytoViva customized ENVI Hyperspectral Image Analysis software. 


\subsection{Manufacture of gold nanoparticles and imaging of nanoparticle-loaded exosomes by Transmission Electron Microscopy (TEM)}

Gold nanoparticles were prepared by mixing of $25 \mathrm{~mL}$ HAuCl4 $(0.5 \mathrm{mM})$ TRIS solution (pH 10) with $25 \mathrm{~mL}$ Pluronic block copolymer F127 (10 mM) solution. The mixture was incubated at $55^{\circ} \mathrm{C}$ for 2 hours and the obtained nanoparticles were separated by centrifugal filtration at $1500 \mathrm{RPM}$ using a filter with $100 \mathrm{kDa}$ cut off. Effective hydrodynamic diameter and zeta-potential of gold nanoparticles were measured by photon correlation spectroscopy using 'ZetaPlus' Zeta Potential Analyzer (Brookhaven Instruments). The average diameter was $10.3 \pm 0.2 \mathrm{~nm}$, the polydispersity index $(P D I)$ value was $0.06 \pm 0.002 \mathrm{~nm}$. For TEM evaluations, a drop of isolated exosomal fraction with incorporated by sonication gold nanoparticles was placed on Formvar®-coated copper grid (150 mesh, Ted Pella Inc., Redding, CA, USA). The dried grid containing exosomes were stained with vanadyl sulfate and visualized using a Philips 201 transmission electron microscope (Philips/FEI Inc., Briarcliff Manor, NY, USA).

\subsection{Poly(lactic-co-glycolic acid) (PLGA) particles preparation}

PLGA nanoparticles were prepared by modification of a w/o/w double emulsion method [61]. Briefly, $3.2 \mathrm{~mL}$ of $5 \%$ polyvinyl alcohol (PVA) was added to $100 \mathrm{~mL}$ of $\mathrm{dH}_{2} \mathrm{O}_{2}$ to form a w/o emulsion. In parallel, $0.35 \mathrm{~g}$ PLGA polymer was dissolved in $3 \mathrm{~mL}$ dichloromethane, and $2 \mu \mathrm{mol}$ DIL was added to the solution. After vigorous stirring, the PLGA emulsion was injected into $50 \mathrm{~mL}$ of $5 \%$ PVA solution under stirring (1500 rpm, at $4^{\circ} \mathrm{C}$ ) to form a primary w/o/w double emulsion. Then, the double emulsion was poured into $500 \mathrm{~mL}$ of deionized water and maintained at $4^{\circ} \mathrm{C}$. In order to evaporate the organic solvent, the temperature was slowly increased up to $20^{\circ} \mathrm{C}$ during two hours. The resulting nanoparticles were centrifuged for 30 minutes at $4000 \mathrm{~g}$, washed with deionized water, and lyophilized. The average particle size measured by Malteasizer DLS was $317.5+1.94 \mathrm{~nm}$ with $P D I$ of 0.113 .

\subsection{Preparation of liposomes}

Liposomes were prepared by reverse phase evaporation method. Briefly $2 \mathrm{mg}$ of phospholipids (95 molar \% of phosphatidyl choline and 5\% of PEG-PE) were dissolved in 6 $\mathrm{ml}$ of chloroform: diisopropyl ether 1:1 mixture. Then $1 \mathrm{ml}$ of $5 \mathrm{mM}$ calcein solution in PBS filtered through $450 \mathrm{~nm}$ syringe filter was added to the mixture. Mixture was intensively vortexed and bath sonicated to form stable emulsion. Organic solvents were evaporated on rotary evaporator forming the liposome aqueous dispersion. 200-250 $\mu \mathrm{l}$ of Millipore water can be added at this point to the mixture in case some part of water was also evaporated. Evaporation was continued to get almost clear dispersion. Then volume was adjusted to $1000 \mu \mathrm{l}$ by addition of small amount of water. Dispersion was vortexed and bath sonicated to get clear solution. Liposomes were sequentially extruded 21 times through $200 \mathrm{~nm}$ polycarbonate filters using a hand extruder (Avanti). Liposomes were purified through a Sepharose CL4B column to remove not encapsulated fluorophore. Volume of the sample was doubled after column separation. Calcein loaded liposomes were used within $24 \mathrm{~h}$ after column separation. 


\subsection{Western blot analysis}

Western blot technique was applied to evaluate loading efficiency for different exoCAT formulations. Protein concentrations were determined using BCA kit (Pierce Biotechnology, Rockford, IL, USA). The catalase protein bands were detected with primary rabbit polyclonal anti-catalase antibodies (Santa Cruse, CA, USA; 1:200 dilution), and secondary horseradish peroxidase (HRP)-conjugated goat anti-rabbit Ig-HRP (Santa Cruse; 1:2500 dilution). The protein bands were visualized by chemiluminescent substrate (Pierce Biotechnology) and quantitated using ImageJ software (NIH, Bethesda, MA, USA) [62]. To correct for loading differences, the levels of proteins were normalized to the constitutively expressed in exosomes TSG101 protein. The TSG101 levels were visualized by TSG101 monoclonal antibodies, Abcam (Cambridge, MA, USA).

\subsection{Enzymatic activity of catalase loaded into exosomes}

The loading efficiency for different exoCAT formulations was assessed by catalase enzymatic activity using hydrogen peroxide decomposition assay [60]. For this purpose, different exoCAT formulations were incubated with pronase $(0.1-0.2 \mathrm{mg} / \mathrm{mL})$ for 3 hours at $37^{\circ} \mathrm{C}$. Following the incubation, the aliquots were subjected for catalytic activity assessment as described above. The same assay was used to examine the preservation of catalase enzymatic activity in exosomes against proteases degradation. Stability of catalase was expressed in the residual activity $v s$. initial activity of catalase.

\subsection{Exosomal uptake in PC12 cells}

PC12 neuronal cells is a common model for in vitro evaluation of drug neuroporotective effects [63]. Exosomes ( $230 \mu \mathrm{g}$ total protein/mL) were subjected to various procedures (incubation at RT, freeze/thaw cycles, or sonication), and then stained with DIL ( $2 \mu \mathrm{mol})$. PC12 cells were seeded into 96-well plate (50,000 cell/well), cultured for three days, and then incubated with different DIL-labeled exoCAT for various times. Following the incubation, the cells were washed three times with ice-cold PBS, and solubilized in Triton X100 (1\%). Fluorescence in each sample was measured by Shimadzu RF5000 fluorescent spectrophotometer $\left(\lambda_{e x}=540 \mathrm{~nm}, \lambda_{e m}=565 \mathrm{~nm}\right)$. The amount of exosomes accumulated in neuronal cells was normalized for the total protein content and expressed as a number of exosomes per $\mathrm{mg}$ of the protein as means \pm S.E.M. $(n=8)$. All exoCAT formulations were prepared at the same level of fluorescence, and a separate calibration curve was used for each exoCAT formulation.

\subsection{In vitro confocal microscopy studies}

Raw 264.7 macrophages $\left(20 \times 10^{6}\right.$ cells/flask) were cultured for three days in DMEM supplemented with $10 \%$ FBS, then concomitant media was collected, and exosomes were isolated by gradient centrifugation as described above. The isolated exosomes $(100 \mu \mathrm{g} / \mathrm{mL}$ total protein) were sonicated, labeled with DIL $(2 \mu \mathrm{mol})$, and incubated with PC12 cells grown on chamber slides $\left(1 \times 10^{5}\right.$ cells/chamber) for various time intervals [64]. Following the incubation period, the cells were washed, fixed, and stained with rabbit anti-PGP9.5 antibodies (green, Abcam, \#10404, 1:500 dilution) for actin micro-filaments, and a fluorescent stain, 4',6-diamidino-2-phenylindole (DAPI) for nuclei prior to the imaging. 
Accumulation of fluorescently-labeled exosomes was visualized by a confocal fluorescence microscopic system ACAS-570 (Meridian Instruments, Okimos, MI, USA) with argon ion laser (excitation wavelength, $488 \mathrm{~nm}$ ) and corresponding filter set. Digital images were obtained using the CCD camera (Photometrics).

\subsection{Amplex Red dye fluorescence assay}

Raw 264.7 macrophages seeded in 96-well plates $\left(0.1 \times 10^{6}\right.$ cells/well $)$ were stimulated with INF- $\gamma(2 \mu \mathrm{g} / \mathrm{mL})$ and LPS $(200 \mathrm{ng} / \mathrm{mL})$ for 4 hours to induce ROS production. Nonactivated cells were used as controls. ExoCAT obtained by sonication in Krebs-Ringer buffer (145 mM NaCl, $4.86 \mathrm{mM} \mathrm{KCl}, 5.5 \mathrm{mM}$ glucose, $5.7 \mathrm{mM} \mathrm{NaH}_{2} \mathrm{PO}_{4}, 0.54 \mathrm{mM} \mathrm{CaCl} 2$, $1.22 \mathrm{mM} \mathrm{MgCl}_{2}, \mathrm{pH}$ 7.4) were supplemented with Amplex Red Dye stock solution (10 $\mathrm{U} / \mathrm{mL}$ HRP and $10 \mathrm{mM}$ Amplex Red), added to the activated macrophages, and the decomposition of ROS was measured by fluorescence at $\lambda_{e x}=563 \mathrm{~nm}, \lambda_{e m}=587 \mathrm{~nm}$ as described [62]. The effect of the same amount of empty exosomes $\left(1.4 \times 10^{11} / \mathrm{mL}\right.$ exosomes), or catalase alone $(3,652 \mathrm{U} / \mathrm{mL})$ on ROS decomposition was evaluated in the control experiments.

\subsection{Cell viability assay}

The protection of PC12 cell by exoCAT prepared by sonication was assessed by MTT assay. For this purpose, PC12 cells $\left(1 \times 10^{5}\right.$ cells $\left./ \mathrm{mL}\right)$ were seeded into a 96-well plate and allowed to attach overnight. Then, the cells were exposed to $200 \mu \mathrm{M}$ 6-OHDA and different exoCAT formulations, or catalase alone, or empty exosomes for four hours. Following the incubation, the cells were washed 3 times with ice-cold PBS, and incubated with the corresponding exoCAT formulations, or catalase alone, or empty exosomes for another 24 hours.

Following the treatment, $20 \mu \mathrm{L}$ MTT $(5 \mathrm{mg} / \mathrm{mL})$ was added into each well. After 3 hours of incubation at $37^{\circ} \mathrm{C}$, the medium containing MTT was removed; and $100 \mu \mathrm{L}$ DMSO was added into each well to dissolve the purple MTT formazan. Absorbances were read at $\lambda=$ $570 \mathrm{~nm}$ by a microplate reader, and cell viability was expressed as a percentage of viable cells in the treated groups compared to the untreated control group.

\subsection{Animals}

C57BL/6 female mice (Charles River Laboratories, Durham, NC, USA) eight weeks of age were treated in strict accordance with the recommendations in the Guide for the Care and Use of Laboratory Animals of the National Institutes of Health. The protocol was approved by the Committee on the Ethics of Animal Experiments of the University of North Carolina at Chapel Hill. All surgery was performed under sodium pentobarbital anesthesia, and all efforts were made to minimize suffering. The animals were kept five per cage with an air filter cover under light-(12-hours light/dark cycle) and temperature-controlled $\left(22 \pm 1^{\circ} \mathrm{C}\right)$ environment. All manipulations with the animals were performed under a sterilized laminar hood. Food and water were given ad libitum.

\subsection{Biodistribution of exosomes in mouse brain with inflammation}

C57BL/6 mice $(n=4)$ were stereotactically injected with 6-OHDA solution $(10 \mu \mathrm{g}$ 6-OHDA in $0.9 \% \mathrm{NaCL}$ with $0.02 \%$ ascorbic acid), flow rate of $0.1 \mu \mathrm{L} / \mathrm{min}$ into the striatum (AP: 
+0.5; L: -2.0 and DV: $-3.0 \mathrm{~mm}$ ) [65]. Twenty one days later (at the peak of inflammation), mice were intranasally (i.n.) or intravenously (i.v.) injected with fluorescently-labeled exosomes $\left(2.4 \times 10^{10}\right.$ exosomes/mouse). Four hours later, mice were sacrificed, perfused, and the brain slides were examined by confocal microscopy. 6-OHDA-intoxicated mice injected with PBS were used as controls. Nuclei were labeled with DAPI. To examine, which type of cells accumulates exosomes in the brain, a co-localization study with cellspecific markers was carried out. For this purpose, the brain slides were co-stained with: primary (1) rabbit polyclonal antibodies to neurons, AntiNeuN (ab128886, Abcam, 1:500 dilution), or (2) anti-tyrosine hydroxylase (TH) rabbit antibodies to TH-neurons (Calbiochem, 1:1000 dilution), or (3) rabbit anti CD146 to endothelial cells (ab75769, Abcam, 1:250 dilution), or (4) rabbit anti-GFAP antibodies to astrocyte marker (ab7260, Abcam, 1:500 dilution), and secondary antibodies, donkey anti-rabbit IgG H\&L Alexa 555 (abcam ab150074, Abcam, 1:500dilution). All slides were permeabilized for $60 \mathrm{~min}$ in $0.1 \mathrm{M}$ citrate buffer pH6.0 and $0.05 \%$ Tween 20, washed 3x 5min with $0.05 \%$ Tween 20 in PBS, blocked for 30 minutes with PBS and 5\% Normal Donkey Serum $+0.05 \%$ Tween 20, and stained with primary antibody at stated dilution overnight at $4 \mathrm{C}$. Following the incubation, slides were washed $3 \times$ 5minutes/wash in PBS/Tween, and stained with secondary antibodies for one hour at room temperature. Then, the slides were washed 3x PBS/Tween 5min/wash ddH2O, and covered using Vectashield Hardset mounting media with Dapi. The images were examined by a confocal fluorescence microscopic system ACAS-570 and corresponding filter set.

\subsection{Immunohistochemical and stereological analyses}

6-OHDA-intoxicated mice $(n=7)$ were treated via i.n. administration with PBS, or catalase alone $\left(1.2 \times 10^{9}\right.$ exosomes with $408.44 \mathrm{U}$ catalase/mouse $\times 2$ in $10 \mu \mathrm{l}$ PBS, ten times every other day), or exoCAT loaded by sonication or saponin permeabilization with the same amount of catalase $\left(1.2 \times 10^{9}\right.$ exosomes/mouse $\times 2$ in $\left.10 \mu \mathrm{PBS}\right)$, or the same amount of empty exosomes 48 hours after the intoxication (10 times every other day). Two control groups of healthy non-intoxicated animals were i.c. injected with PBS, and then 48 hours later were i.n. injected with PBS, or empty exosomes. Twenty one days later, animals were sacrificed, perfused; brains were removed, washed, post-fixed, and immunohistochemical analysis was performed in $30 \mu \mathrm{m}$ thick consecutive coronal brain sections as described [46]. For the detection of microglia activation, tissue sections were incubated with primary monoclonal rat anti-mouse anti-CD11b antibodies (1:500 dilution), and secondary biotinylated goat anti-rat antibodies (Vector Laboratories, Burlingame, CA, 1:200 dilution). Thus, activated microglia within the $S N p c$ will exhibit a more amoeboid morphology with sent out branches, compared to ramified barely visible resting microglia. In addition, levels of astrocytosis were assessed in the ventral midbrain region by fluorescent analysis of glial fibrillary acidic protein (GFAP) expression. For the GFAP staining, tissue sections were permeabilized with $0.01 \%$ Triton X-100 in TBS for 30 minutes and blocked for 1 hour with $10 \%$ normal goat serum (NGS, Vector Laboratories Inc., Burlingame, CA), then incubated with rabbit anti-GFAP primary polyclonal antibodies ab7260 (AbCam, Cambridge, MA) 1:100 dilution for 16 hours at $4^{\circ} \mathrm{C}$. Tissue slides were incubated with goat anti-rabbit Alexa Fluor 647 secondary antibodies (Invitrogen; 1:200 dilution) for 1 hour, and mounted on slides. Immunoreactivity was evaluated by fluorescent analysis using confocal microscope 
Zeiss 510 Meta Confocal Laser Scanning Microscope (Jena, Germany), and ImageJ software (NIH, Bethesda, MA, USA). For the assessment of neuroprotective effects, a TH staining was used to quantitate numbers of DA neurons [66]. The total number of TH-positive DA neurons was counted by using the optical fractionator module in StereoInvestigator software (MicroBrightField, Inc., Williston, VT) [46].

\subsection{Apomorphine Test}

C57BL/6 mice were i.c. injected with 6-OHDA $(n=7)$. Healthy mice i.c. injected with PBS were used as a control [67]. Starting from 48 hours after intoxication, mice were i.n. injected with PBS, or exoCAT obtained by saponin permeabilization every other day for two weeks. Twenty one days later, the animals were injected with apomorphine $(0.05 \mathrm{mg} / \mathrm{kg}$, s.c. $)$ and rotations were scored every $10 \mathrm{~min}$ for $90 \mathrm{~min}$ as described [68].

\subsection{Statistical analysis}

For the all experiments, data are presented as the mean \pm S.E.M. Tests for significant differences between the groups were performed using a t-test or one-way ANOVA with multiple comparisons (Fisher's pairwise comparisons) using GraphPad Prism 5.0 (GraphPad software, San Diego, CA, USA). A minimum $p$ value of 0.05 was chosen as the significance level.

\section{RESULTS}

\subsection{Manufacture of exosomal formulations of catalase (exoCAT)}

Catalase was incorporated into exosomes using different methods: a) incubation at RT with or without of saponin permeabilization; b) freeze/thaw cycles; c) sonication, and d) extrusion procedures. The last two methods were utilized to cause a reformation/ deformation of exosomes in the presence of catalase. The obtained exoCAT formulations were purified from non-incorporated catalase by a gel-filtration chromatography with Sepharose 6BCL as described in Materials and Methods section.

According to the western blot analysis, the amount of catalase loaded into exosomes increased in the row: the incubation at $\mathrm{RT}<$ freeze/thaw cycle $<$ sonication $\approx$ extrusion (Fig. 1 A). These results were confirmed by catalytic activity of the enzyme (Fig. 1 B). ExoCAT obtained by sonication and extrusion showed the highest catalytic activity, followed by exoCAT obtained by freeze/thaw cycles, and then the incubation at RT (without saponin permeabilization). The incubation of catalase with exosomes at RT resulted in the lowest loading efficiency $(4.9 \pm 0.5 \%, n=4)$ among all evaluated methods. Nevertheless, when exosomes were permeabilized with saponin, catalase loading efficiency significantly increased $(18.5 \pm 1.3 \%, p<0.05)$. Furthermore, sonication and extrusion procedures resulted in the most efficient enzyme incorporation $(26.1 \pm 1.2 \%$ and $22.2 \pm 3.1 \%$, respectively). We hypothesized that a formation of transient pores or even reformation of exosomes upon sonication and extrusion allowed diffusion of catalase from the surrounding media into exosomes. The freeze/thaw cycles technique gave somewhat intermediate values of loading efficiency $(14.7 \pm 1.1 \%)$. Regarding the yield of the catalase formulations, about $98 \%$ of exosomes were recovered upon all loading methods. Noteworthy, catalase structure 
was stabilized upon incorporation into exosomes. No loss of catalase enzymatic activity was detected in exoCAT upon sonication, while catalase alone was significantly deactivated in the same conditions (down to $14 \%$ ).

The release of catalase from pre-loaded exosomes was evaluated by enzymatic activity using dialysis membranes with a cut off $2000 \mathrm{KDa}$ (Fig. 1 C). The fastest release was observed for the formulation obtained by incubation at RT without saponin (Fig. 1 C). In contrast, a prolonged and sustained release was recorded for exoCAT obtained by sonication; less than $40 \%$ of catalase was released over 24 hours. Taken together, the sonication of exosomes in the presence of catalase resulted in the high loading efficiency, and sustained release of enzymatically-active catalase. In particular, each mg of exoCAT obtained by sonication contained approximately $1,376 \pm 64.1 \mathrm{U}$ of catalase activity in $4 \times 10^{11}$ exosomes $/ \mathrm{mL}$.

\subsection{Characterization of exoCAT formulations}

The size of catalase-loaded exosomes was determined by DLS and NTA techniques (Table 1). Particles of naked catalase showed small size $(9.5 \mathrm{~nm})$ with low polydispersity index $(P d I)$, which was close to the theoretical diameter $(10.5 \mathrm{~nm})$ of a single protein globule calculated from the molecular mass of the enzyme [69]. An average diameter of empty exosomes was around $100 \mathrm{~nm}$ with a relatively high polydispersity (Table 1). The incubation of catalase with exosomes at RT (with or without saponin) did not significantly alter their size. However, freeze/thaw cycles, extrusion, and sonication of exosomes resulted in the significant size increases, especially, in the presence of catalase (Table 1). Overall, both DLS and NTA analyses indicate the size of the obtained exoCAT formulations was in the range of $100-200 \mathrm{~nm}$. This suggests that the relatively small catalase-loaded nanoparticles might enter the target cells by endocytosis. We will investigate the intracellular trafficking of exoCAT in our further studies.

Based on the data from NTA experiments and evaluations of catalase activity, about $940 \pm$ 15 catalase molecules per exosome were incorporated by sonication. Noteworthy, exosomal formulations were stable at RT over a week; no changes in size and/or catalase activity were recorded. Furthermore, the restitution of lyophilized exoCAT in water solutions did not alter the size, and activity of exosomal formulations.

The AFM images revealed considerable differences in the morphology of exoCAT formulations (Fig. 1 D). Naïve exosomes isolated from Raw 264.7 macrophages, as well as exosomes incubated with catalase at RT, or obtained by extrusion procedures have a round morphology. Particles of exoCAT formulation manufactured by freeze-thaw cycle were considerably larger in size consisting of several dozen of smaller exosomes, probably due to the aggregation. The AFM images of sonicated exoCAT demonstrated non-spherical associates with a variety of shapes $($ Fig. 1 D).

Next, the loading of catalase into exosomes was confirmed by Hyperspectral microscopy. This technique enables optical observation, spectral characterization, and mapping of nanoscale materials in a wide range of biological and material-based environments.

Supplemental Fig. S1 shows representative hyperspectral images of exosomes loaded with catalase by sonication (Fig. S1 A), and empty exosomes sonicated at the same conditions 
(Fig. S1 B). The measurements illustrate a narrowing of the spectral response of the loaded versus the unloaded exosomes (Fig. S1 C). This suggests that significant alterations/ perturbations occur upon catalase incorporation into exosomes. The incorporation of catalase into exosomes significantly improved its enzymatic stability against protease degradation (Fig. 2). In accordance with the loading efficiency (Fig. 1 A-B), and release kinetics (Fig. 1 C), the stability of catalase was increased in the row: incubation at RT without saponin < freeze/thaw cycles < sonication (Fig. 2). Altogether, this indicates that catalase was not only associated with the surface of exosome, but was also incorporated into them.

\subsection{Accumulation and therapeutic efficacy of exoCAT in target neuronal cells in vitro}

The ability of nanocarriers to deliver the drug payload into target cells is crucial for the therapeutic efficiency of exosomal formulations. First, we examined, whether manipulations with exosomes affect their transport into target cells in vitro. Exosomes were subjected to various loading procedures (incubation at RT, freeze/thaw cycles, or sonication), labeled with lipophilic fluorescent dye, DIL, and then incubated with PC12 cells. Striking differences in accumulation levels were recorded (Fig. $3 \mathbf{A}$ ). In spite of their large size, sonicated exosomes were taken up at considerably greater levels than those subjected to freeze/thaw cycles, or incubation at RT. This suggests superior interactions of sonicated exosomes than other exosomal preparations with cellular plasma membranes. We speculate that a reorganization of exosomes upon sonication may result in the exposure of hydrophobic parts of the cellular lipid bilayers or incorporated proteins that improve their interactions with plasma membranes of target cells. Next, confocal images confirmed a profound accumulation of sonicated DIL-labeled exosomes in PC12 cells (Fig. 3 B). The exosomes were efficiently internalized into neurons followed 3-hour incubation (Fig. 3 B-I), filled the entire neuronal body and build up on the plasma membranes at later time points (Fig. 3 B-II). The unparalleled accumulation of exosomes in target cells was even more evident in comparison with the considerably lower uptake of commonly used for the drug delivery polymer-based PLGA nanoparticles (100 nm, Fig. 3 B-III), or liposomes (Fig. 3 BIV) that were applied at the same level of fluorescence. These nanocarriers (PLGA nanoparticles and liposomes) were utilized for the brain delivery of L-DOPA to treat PD $[70,71]$.

Given the efficient accumulation of exosomes in PC12 neurons, we hypothesized that exosomes with incorporated catalase may deliver a substantial amount of the enzyme into target neuronal cells, and as a result, provide a superior neuro-protective activity against oxidative stress. To test this hypothesis, the effect of exoCAT formulations on neuronal survival was evaluated in PC12 cells pre-treated with 6-OHDA to recapitulate components of PD neurodegeneration in vitro. Following the 6-OHDA pre-treatment, the PC12 cells were supplemented with various exoCAT formulations, and the neuronal survival was evaluated by MTT test (Fig. 3 C). As expected, exoCAT prepared by sonication and extrusion demonstrated greater neuroprotective activity than exosomes loaded with catalase by freeze/thaw cycles or incubation at RT, probably, due to the facilitated exoCAT accumulation, and/or protection of catalase against degradation in exosomal carriers. Noteworthy, permeabilization of exosomes by saponin upon loading with catalase 
significantly increased neuroprotection effect produced by this formulation (Fig. 3 C).

Catalase alone, as well as empty exosomes did not protect PC12 cells at these experimental conditions.

Next, exoCAT obtained by sonication was evaluated for the ability to eliminate ROS produced by activated macrophages in vitro (Fig. $3 \mathrm{D})$. In this experiment, macrophages pre-incubated with a mixture of LPS $(100 \mathrm{ng} / \mathrm{mL})$ and TNF-a $(100 \mathrm{ng} / \mathrm{mL})$ were utilized as a model of activated microglia in the PD brain. The activation of macrophages resulted in the significant increase in ROS production, whereas the addition of exoCAT considerably decreased hydrogen peroxide levels (Fig. 3 D). Interesting, empty exosomes also decreased ROS levels in activated macrophages down to the levels of the control non-activated cells, although this effect was less significant than the effect of exoCAT. This indicates that exosomes by themselves (probably, their internal content) have a capacity to deactivate free radicals. This result is consistent with the studies demonstrating protection effects of exosomes against myocardial ischemia/reperfusion injury [42]. Altogether, these data suggests that exoCAT can be a useful tool for ROS deactivation and neuronal protection against oxidative stress.

\subsection{Transport of exosomes into mouse brain with inflammation}

To visualize the ability of exosomes to target and deliver their payload to inflamed brain tissues, confocal imaging studies were conducted in a PD mouse model. To induce brain inflammation, C57BL/6 mice were intracranially (i.c.) injected with 6-OHDA into SNpc. Twenty one days later (at the pick of inflammation), mice were injected with DIL-labeled exosomes $\left(2.4 \times 10^{10}\right.$ exosomes $/ 20 \mu \mathrm{L} /$ mouse) through intranasal (i.n.) $($ Fig. 4 B $)$, or intravenous (i.v.) (Fig. 4 C) routs. Mice i.n. injected with PBS were used as controls (Fig. 4 D). Four hours later, mice were euthanized, perfused, and brain slides were examined by confocal microscopy. Nuclei were stained with DAPI (blue). The images revealed a wide distribution of exosomes throughout the brain, in particular, cerebral frontal cortex, central sulcus, and cerebellum (Fig. 4 A). The amount of exosomes delivered upon the i.n. administration was greater (Fig. 4 B) than those delivered through i.v. injection (Fig. 4 C). Confocal images showed diffuse fluorescent staining throughout the brain tissues along with the stained vesicular compartments localized predominantly in perinuclear regions (shown by arrows). No fluorescence was found in control mice with the PBS injection (Fig. 4 D). As such, i.n. route of administration was selected for the further evaluations of exoCAT therapeutic effects.

\subsection{Catalase-loaded exosomes protect SNpc neurons against oxidative stress in mice with acute brain inflammation}

The neuropathology of PD includes brain inflammation, microglial activation, and secretion of neurotoxins, such as ROS, all of which contribute to neurodegeneration and degradation of motor function [72]. We posit that the successful brain delivery of catalase will reduce neuroinflammation resulting in improved disease outcomes. For this purpose, C57BL/6 mice were stereotactically injected with 6-OHDA into $S N p c$, and 48 hours later, mice were i.n. injected with exoCAT $\left(1.2 \times 10^{9}\right.$ exosomes with catalase activity $408.44 \mathrm{U} / 10 \mu \mathrm{L} /$ mouse into each nostril, 10 times every other day), or the same amount of catalase alone. Two 
exoCAT formulations were evaluated; catalase-loaded exosomes by sonication, or permeabilization with saponin at RT ( $n=7)$. Mice intoxicated with 6-OHDA, and then i.n. injected with PBS were used as control animals with brain inflammation (PD mice). Nonintoxicated animals i.c. injected with PBS were used as healthy controls. To evaluate the effect of empty exosomes in healthy brain, another control group of healthy animals was i.c. injected with PBS, and then empty exosomes. Twenty-one days following administration of the test formulation, the mice were sacrificed, perfused with paraformaldehide (PFA), and brains harvested. Brain slides were stained with antibodies to activated microglia (Fig. 5), astrocytes (Supplemental Fig. S2), or TH-expressing DA neurons (Fig. 6). The quantification of the obtained results for anti-inflammatory and neuroprotective effects is presented on Fig. $5 \mathbf{F}$ and $6 \mathbf{F}$, respectively.

6-OHDA injections produced substantial brain inflammation signified by up-regulated expression of CD11b by microglia within the $S N p c$ that exhibited a more amoeboid morphology (Fig. 5 C) than ramified microglia in PBS-treated mice (Fig. 5 A). Furthermore, the 6-OHDA-mediated intoxication resulted in the complete degeneration of DA neurons in the ipsilateral hemisphere $S N p c$ (Fig. 6 C, arrow). The anti-inflammatory effect of exoCAT was demonstrated by the significant $(p<0.005)$ reduction in microgliosis as measured by CD11b expression (Fig. 5 D, E, G) and decrease in astrocytosis as demonstrated by GFAP levels (Supplemental Fig. S2). Furthermore, i.n. administration of exoCAT resulted in a 3fold increase in survived DA neurons (Fig. 6 D, E, G) compared to the control 6-OHDAtreated mice injected with PBS (Fig. 6 C, G). The neuroprotection was greater in the animal group treated with exoCAT obtained by permeabilization with saponin (Fig. 6 E, G) than with exoCAT loaded by sonication (Fig. 6 D, G). Not surprisingly, free catalase was not effective in decreasing inflammation or providing neuroprotection in 6-OHDA-intoxicated mice (Fig. 5 E and 6 E).

Next, we investigated the effect of exoCAT formulation obtained by saponin permeabilization in apomorphine test. Mice intoxicated with 6-OHDA and treated i.n. with PBS showed $150 \pm 11$ rotations per minute. In contrast, mice intoxicated with 6-OHDA and then treated i.n. with exoCAT as described above have significantly less rotations (26.1 \pm 3.1 per minute). Noteworthy, non-intoxicated healthy controls did not rotate at all.

Finally, we investigated possible toxic effect of exosomal carriers alone. No effect of empty exosomes on the microglial activation or number of DA neurons was found in healthy mice (Fig. $5 \mathrm{~B}$ and $6 \mathrm{~B}$ ) compared to the healthy PBS-treated controls (Fig. $5 \mathrm{~A}$ and $6 \mathrm{~A}$, respectively) suggesting absence of neurotoxic effects of exosomal carriers in the brain. This result was confirmed in in vitro model of primary cortical or DA neurons isolated from mouse pups. Thus, a 48-hour exposure of primary neurons to exosomes released from macrophages did not affect neuronal survival (Supplemental Fig. S3). Overall, these results demonstrate that exosomal formulations of catalase may be useful for PD therapies.

\subsection{Co-localization of exosomes with various types of cells in the brain}

To assess, which cell type in the brain accumulates exosomes, brain slides were co-stained with different cell markers (Fig. 7). Interesting, exosomes were mostly co-localized with 
neurons, microglia and partially with endothelial cells (Fig. 7). Noteworthy, along with distinct endosomal compartments filled with exosomes, a diffuse exosomal staining was evident throughout all brain tissues. It is likely the exosome-mediated delivery of catalase to activated microglia, astrocytes, and neurons in the inflamed brain may result in ROS degradation and neuroprotection in PD patients.

\section{DISCUSSION}

Currently, there are no treatments that halt or reverse the course of $\mathrm{PD}$, and only palliative therapies, such as replacement strategies for missing neurotransmitters, exist. The inability of most potent therapeutics, and especially therapeutic proteins, to cross the BBB following systemic administration dictates the necessity to develop unconventional, clinically applicable drug delivery systems. In this respect, biocompatible vehicles, such as exosomes, may help to solve this challenging task. Recent findings indicate that communications mediated via exosomes is a common mechanism in the CNS [73]. Therefore, several investigations suggested harnessing this natural mechanism of protein and genetic material transfer for the delivery of different therapeutics [20, 33-39, 74].

Oxidative stress has long been implicated in the process of neurodegeneration seen in Parkinson's patients. Indeed, the pathogenesis of PD is associated with a lack of the natural antioxidants catalase, glutathione and superoxide dismutase in the midbrain region, specifically, in the $S N p c$ [75]. It was demonstrated that cells of the immune system, and particularly microglia, release pro-inflammatory cytokines in response to different stress conditions [76] leading to neuronal demise [77]. Antioxidants can inhibit inflammatory responses and protect dopaminergic neurons as reported in laboratory and animal models of PD [78-84]. In particular, catalase, one of the most efficient antioxidants found in nature, has been shown to rescue primary cerebellar granule cells in in vitro models of PD [85, 86]. Several clinical studies have assessed the therapeutic efficacy of low molecular antioxidants for PD therapy. Unfortunately, the results of these investigations have been disappointing [87] due to the drug poor pharmacokinetics and inability to penetrate the BBB. Thus, the development of novel approaches for brain delivery of antioxidants, and in particular redox enzymes, is of utmost importance.

We report here the development of a new exosomal-based technology for catalase CNS delivery to treat PD. Catalase is a large protein (MW $240 \mathrm{~K}$ ) that is susceptible to deactivation and rapid degradation. Therefore, different techniques for catalase loading into exosomes were evaluated: incubation at RT with or without saponin permeabilization, freeze/thaw cycles, sonication, or extrusion. Our data indicate the size, morphology, loading efficiency, and stability of exoCAT formulations strongly depended on the method of preparation. Thus, the extensive reformation and reshaping of exosomes upon sonication and extrusion enabled catalase diffusion across relatively tight and highly structured lipid bilayers and resulted in the high loading efficiency of exosomal carriers. Notably, these approaches for incorporation into exosomes are not specific only for proteins, but can be applied to other therapeutic and imaging agents. Thus, TEM studies indicated that a substantial amount of gold nanoparticles with the diameter $(10.3 \pm 1.2 \mathrm{~nm})$ can be also incorporated/associated with exosomes by sonication (Supplemental Fig. S4). Interesting, 
similar to the sonicated exoCAT (Fig. 1D), nanoparticles-loaded exosomes showed nonspherical morphology with a variety of shapes (Supplemental Fig. S4). Indeed, it should be taken into consideration that the disruption of the exosomes integrity during sonication or extrusion procedures may alter their immune-privileged status, and therefore, make them visible for the MPS. We will investigate all benefits and disadvantages of these manipulations in subsequent studies.

Noteworthy, saponin treatment also increased loading of catalase into exosomes. Saponin is the efficient permeabilization agent for cellular plasma membranes [88]. We hypothesized that similar to the whole cells, saponin may selectively remove membrane-bound cholesterol of exosomes, creating holes/pores in the exosomal lipid bilayers and therefore, promoting catalase incorporation. Overall, exoCAT obtained by sonication and extrusion, as well as saponin treatment showed the high loading efficiency, preservation of catalase enzymatic activity against proteases degradation, and prolonged and sustained release.

Regarding the delivery of incorporated therapeutics to the target cells, this study demonstrated the extraordinary ability of exosomes to interact with target cells and deliver their "payload" into neighboring neurons. Confocal images revealed fluorescently-labeled exosomes were adhering and overflow neuronal cells in abundance. Indeed, comprising of cellular membranes exosomes should have an exceptional ability to interact with target cells. Furthermore, exosomal surface is rich with tetraspanins and integrins [24, 31, 89] that enable the efficient attachment to the plasma membrane of target cells. As a result, exosomes accumulated in considerably greater levels in PC12 cells than PLGA nanoparticles that have been used as common nanocarriers for PD therapy [90] or liposomes [71, 91]. Interesting, the accumulation levels varied for different exosomal preparations. In particular, sonicated exosomes showed the greatest uptake in neurons compared to exosomes incubated at RT, or aggregates obtained by freeze/thaw cycles. We hypothesized that a reorganization of exosomes upon sonication may alter the content of surface proteins as well as organization of lipid bilayers that resulted in the increased exosomal interactions with cellular membranes of target cells. Obviously, this effect may play a positive role and overpower negative effects of decreased uniformity and increased visibility of exosomal carriers for the cells of immune system mentioned above.

Concerning the antioxidant activity, exoCAT formulations showed the efficient ROS deactivation and significant neuroprotective effects against oxidative stress in vitro. In accordance with the loading efficiency, exoCAT obtained by sonication and extrusion provided the most potent neuroprotection. We reported earlier that exosomes secreted from preloaded with nanoformulated catalase macrophages were accumulated in adjacent cells diffusing broadly throughout the cytoplasm and avoiding degradation in lysosomes [43]. This mechanism enabled the drug to reach different intracellular compartments, such as mitochondria, and endoplasmic reticulum, and produce potent therapeutic effects [43]. We speculated that the same favorable intracellular localization of exoCAT may support the superior antioxidant and protective activity of exoCAT in neurons. Further investigations will reveal mechanism and intracellular trafficking of different exosomal formulations in target cells. 
The intranasal administration provides two main routes for the CNS drug delivery: a) transport across the single epithelial cell layer directly to the systemic blood circulation without first-pass hepatic and intestinal metabolism; and b) transport along the olfactory nerve cells, when drug can bypass the BBB and enter the brain directly. Furthermore, this route is attractive due to the possibility of non-invasive multiple treatments with high patient compliancy. Interesting, some investigators hypothesized that PD have its origin in the bulbs olfactory [92]. Subsequently, it could spread, ascending cell-by-cell through brainstem, midbrain, and other regions of the brain, and finally result in PD. Therefore, we reasoned that intranasal administration of exoCAT may work the same way delivering therapeutic catalase to the affected brain areas. We report here that intranasally administered exosomes diffused through the mouse brain, localizing predominantly in the cerebral frontal cortex, central sulcus, and cerebellum. This result is consistent with previously reported findings regarding the intranasal administration of exosomes loaded with anti-inflammatory drug, curcumin [36].

The most important finding of our investigation is that selected exoCAT formulations significantly decreased brain inflammation and increased neuronal survival in a PD mouse model. The mechanism of these effects is yet to be uncovered. We hypothesized the encapsulation of catalase into exosomes may preserve catalase enzymatic activity, prolong the blood circulation time, reduce immunogenicity, and improve its interaction with epithelial cells, thus improving drug transport and therapeutic effects in PD. Here, we investigated two exosomal formulations that were obtained by saponin treatment, and sonication. These formulations were chosen as the most efficient ones that can provide high loading and sustained drug release. In addition, we evaluated, whether the reformation of exosomes upon sonication affected their therapeutic efficacy in vivo. We demonstrated that both formulations significantly decreased neuroinflammation and provided potent neuroprotection in 6-OHDA mouse model. Furthermore, catalase-loaded exosomes obtained by permeabilization with saponin have superior therapeutic effects than those obtained by sonication. It is likely that exoCAT obtained by permeabilization with saponin might have better uniformity in their surface morphology presumably with the intact membrane proteins. This may lead to a lower visibility for RES and clearance by macrophages. Overall, successful development of exosome-mediated delivery of catalase could lead to a viable therapy for patients with PD.

In clinical settings, different approaches may be applied to introduce exosomal-based drug delivery systems (Fig. 8). First, exosomal carriers harvested from peripheral blood monocytes by apheresis will be loaded with a therapeutic agent and readministered back into the patient. As an alternative approach, stem cells may be harvested from bone marrow, propagated in culture to obtain specific cell types, or even subtypes, and then released naïve exosomes will be loaded with a therapeutic agent. Although this approach would require a more invasive procedure, a significant amount as well as storage of well-characterized exosomal carriers will be possible [93]. Noteworthy, exosomes can be concentrated, lyophilized, and reconstituted in water solutions, as this study demonstrated. This will allow scalability, standardization, and consistency of manufacturing different lots of exososomal drug formulations, when a considerable amount and long-turn storage of exosomes might be 
required. Finally, a library of various types of exosomal carriers for different drug formulations could be developed in future and stored in stock for emergency situations.

Also of note, further tailoring exosomes can provide biologically-active carriers that may be modified in accordance to the disease and produce cytotoxic (for a cancer treatment) or neuroprotective (for the treatment of neurodegenerative disorders) effects enhancing the therapeutic outcomes. Thus, drug-loaded exosomes may well serve as a next generation drug delivery mechanism that combines nanoparticle size with non-cytotoxic effects, a high drug carrying capacity, and a low immunogenic profile.

\section{CONCLUSION}

This work demonstrates that exosomes are the exceptionally potent carriers for therapeutic protein, catalase. We developed an efficient method of the drug loading into exosomes without significantly altering their structure, and showed that exosomes loaded with catalase efficiently accumulate in neurons and microglial cells in the brain and produce a potent neuroprotective effect. These findings indicate that an exosomal based formulations could be a valuable tool in the future for the therapy of neurodegenerative disorders. Of course, the complexity of these interventions is challenging, yet they promise an unparalleled efficacy in the treatment of many life-threatening conditions, including those lacking effective pharmacotherapy. Moreover, a positive outcome would also suggest the more general applicability of this innovative approach for delivering therapeutics to the central nervous system (CNS) and beyond.

\section{Supplementary Material}

Refer to Web version on PubMed Central for supplementary material.

\section{ACKNOWLEDGMENTS}

This study was supported by the United States National Institutes of Health grants 1RO1 NS057748 (to EVB), RR021937 (to AVK), and Ministry of Education and Science of Russian Federation grants 11.G34.31.0004, 02.740.11.5232, and RSF-14-13-00731 (both to AVK and NLK). We are grateful for the assistance during Hyperspectral Microscopy experiments provided by the CytoViva company staff. The authors wish to thank Carrie Donley of the Chapel Hill Analytical and Nanofabrication Laboratory (CHANL) in the Department of Applied Physical Sciences at the University of North Carolina at Chapel Hill for the acquisition of AFM images.

\section{REFERENCES}

1. Mcgeer PL, Itagaki S, Boyes BE, Mcgeer EG. Reactive microglia are positive for HLA-DR in the substantia nigra of Parkinson's and Alzheimer's disease brains. Neurology. 1988; 38(8):1285-1291. [PubMed: 3399080]

2. Busciglio J, Yankner BA. Apoptosis and increased generation of reactive oxygen species in Down's syndrome neurons in vitro. Nature. 1995; 378(6559):776-779. [PubMed: 8524410]

3. Ebadi M, Srinivasan SK, Baxi MD. Oxidative stress and antioxidant therapy in Parkinson's disease. Prog Neurobiol. 1996; 48(1):1-19. [PubMed: 8830346]

4. Wu DC, Teismann P, Tieu K, et al. NADPH oxidase mediates oxidative stress in the 1-methyl-4phenyl-1,2,3,6-tetrahydropyridine model of Parkinson's disease. Proc Natl Acad Sci U S A. 2003; 100(10):6145-6150. [PubMed: 12721370]

5. Ambani LM, Van Woert MH, Murphy S. Brain peroxidase and catalase in Parkinson disease. Arch Neurol. 1975; 32(2):114-118. [PubMed: 1122174] 
6. Riederer P, Sofic E, Rausch WD, et al. Transition metals, ferritin, glutathione, and ascorbic acid in parkinsonian brains. J Neurochem. 1989; 52(2):515-520. [PubMed: 2911028]

7. Abraham S, Soundararajan CC, Vivekanandhan S, Behari M. Erythrocyte antioxidant enzymes in Parkinson's disease. Indian J Med Res. 2005; 121(2):111-115. [PubMed: 15756044]

8. Pardridge WM. Drug transport across the blood-brain barrier. J Cereb Blood Flow Metab. 2012; 32(11):1959-1972. [PubMed: 22929442]

9. Kabanov, AV.; Batrakova, EV. Polymer nanomaterials. In: Gendelman, HE.; Ikezu, T., editors. Neuroimmune Pharmacology. Springer; Omaha: 2008. p. 691-707.

10. Silva GA. Nanotechnology applications and approaches for neuroregeneration and drug delivery to the central nervous system. Ann N Y Acad Sci. 2010; 1199:221-230. [PubMed: 20633128]

11. Peng Q, Zhang S, Yang Q, et al. Preformed albumin corona, a protective coating for nanoparticles based drug delivery system. Biomaterials. 2013

12. Beckman JS, Minor RL Jr. White CW, Repine JE, Rosen GM, Freeman BA. Superoxide dismutase and catalase conjugated to polyethylene glycol increases endothelial enzyme activity and oxidant resistance. J Biol Chem. 1988; 263(14):6884-6892. [PubMed: 3129432]

13. Yoshida K, Burton GF, Mckinney JS, Young H, Ellis EF. Brain and tissue distribution of polyethylene glycol-conjugated superoxide dismutase in rats. Stroke. 1992; 23(6):865-869. [PubMed: 1595107]

14. Veronese FM, Caliceti P, Schiavon O, Sergi M. Polyethylene glycol-superoxide dismutase, a conjugate in search of exploitation. Adv Drug Deliv Rev. 2002; 54(4):587-606. [PubMed: 12052716]

15. Dams ET, Laverman P, Oyen WJ, et al. Accelerated blood clearance and altered biodistribution of repeated injections of sterically stabilized liposomes. J Pharmacol Exp Ther. 2000; 292(3):10711079. [PubMed: 10688625]

16. Ishida T, Maeda R, Ichihara M, Irimura K, Kiwada H. Accelerated clearance of PEGylated liposomes in rats after repeated injections. J Control Release. 2003; 88(1):35-42. [PubMed: 12586501]

17. Ishida T, Kashima S, Kiwada H. The contribution of phagocytic activity of liver macrophages to the accelerated blood clearance (ABC) phenomenon of PEGylated liposomes in rats. J Control Release. 2008; 126(2):162-165. [PubMed: 18160170]

18. Armstrong JK, Hempel G, Koling S, et al. Antibody against poly(ethylene glycol) adversely affects PEG-asparaginase therapy in acute lymphoblastic leukemia patients. Cancer. 2007; 110(1):103111. [PubMed: 17516438]

19. Garratty G. Modulating the red cell membrane to produce universal/stealth donor red cells suitable for transfusion. Vox sanguinis. 2008; 94(2):87-95. [PubMed: 18034787]

20. Lai RC, Yeo RW, Tan KH, Lim SK. Exosomes for drug delivery - a novel application for the mesenchymal stem cell. Biotechnology advances. 2012

21. Johnsen KB, Gudbergsson JM, Skov MN, Pilgaard L, Moos T, Duroux M. A comprehensive overview of exosomes as drug delivery vehicles - endogenous nanocarriers for targeted cancer therapy. Biochim Biophys Acta. 2014; 1846(1):75-87. [PubMed: 24747178]

22. Tian Y, Li S, Song J, et al. A doxorubicin delivery platform using engineered natural membrane vesicle exosomes for targeted tumor therapy. Biomaterials. 2014; 35(7):2383-2390. [PubMed: 24345736]

23. Vlassov AV, Magdaleno S, Setterquist R, Conrad R. Exosomes: current knowledge of their composition, biological functions, and diagnostic and therapeutic potentials. Biochim Biophys Acta. 2012; 1820(7):940-948. [PubMed: 22503788]

24. Thery C, Amigorena S, Raposo G, Clayton A. Isolation and characterization of exosomes from cell culture supernatants and biological fluids. Curr Protoc Cell Biol. 2006; 22 Chapter 3, Unit 3.

25. Bhatnagar S, Shinagawa K, Castellino FJ, Schorey JS. Exosomes released from macrophages infected with intracellular pathogens stimulate a proinflammatory response in vitro and in vivo. Blood. 2007; 110(9):3234-3244. [PubMed: 17666571]

26. Clayton A, Turkes A, Navabi H, Mason MD, Tabi Z. Induction of heat shock proteins in B-cell exosomes. J Cell Sci. 2005; 118:3631-3638. Pt 16. [PubMed: 16046478] 
27. Nolte-'T Hoen EN, Buschow SI, Anderton SM, Stoorvogel W, Wauben MH. Activated T cells recruit exosomes secreted by dendritic cells via LFA-1. Blood. 2009; 113(9):1977-1981. [PubMed: 19064723]

28. Johnstone RM. The Jeanne Manery-Fisher Memorial Lecture 1991. Maturation of reticulocytes: formation of exosomes as a mechanism for shedding membrane proteins. Biochem Cell Biol. 1992; 70(3-4):179-190. [PubMed: 1515120]

29. Zomer A, Vendrig T, Hopmans ES, Van Eijndhoven M, Middeldorp JM, Pegtel DM. Exosomes: Fit to deliver small RNA. Commun Integr Biol. 2010; 3(5):447-450. [PubMed: 21057637]

30. Valadi H, Ekstrom K, Bossios A, Sjostrand M, Lee JJ, Lotvall JO. Exosome-mediated transfer of mRNAs and microRNAs is a novel mechanism of genetic exchange between cells. Nat Cell Biol. 2007; 9(6):654-659. [PubMed: 17486113]

31. Thery C, Ostrowski M, Segura E. Membrane vesicles as conveyors of immune responses. Nat Rev Immunol. 2009; 9(8):581-593. [PubMed: 19498381]

32. Skog J, Wurdinger T, Van Rijn S, et al. Glioblastoma microvesicles transport RNA and proteins that promote tumour growth and provide diagnostic biomarkers. Nat Cell Biol. 2008; 10(12): 1470-1476. [PubMed: 19011622]

33. Alvarez-Erviti L, Seow Y, Yin H, Betts C, Lakhal S, Wood MJ. Delivery of siRNA to the mouse brain by systemic injection of targeted exosomes. Nat Biotechnol. 2011; 29(4):341-345. [PubMed: 21423189]

34. Lakhal S, Wood MJ. Exosome nanotechnology: an emerging paradigm shift in drug delivery: exploitation of exosome nanovesicles for systemic in vivo delivery of RNAi heralds new horizons for drug delivery across biological barriers. Bioessays. 2011; 33(10):737-741. [PubMed: 21932222]

35. Van Den Boorn JG, Schlee M, Coch C, Hartmann G. SiRNA delivery with exosome nanoparticles. Nat Biotechnol. 2011; 29(4):325-326. [PubMed: 21478846]

36. Zhuang X, Xiang X, Grizzle W, et al. Treatment of Brain Inflammatory Diseases by Delivering Exosome Encapsulated Anti-inflammatory Drugs From the Nasal Region to the Brain. Mol Ther. 2011; 19(10):1769-1779. [PubMed: 21915101]

37. Lai CP, Breakefield XO. Role of exosomes/microvesicles in the nervous system and use in emerging therapies. Frontiers in physiology. 2012; 3:228. [PubMed: 22754538]

38. El Andaloussi S, Lakhal S, Mager I, Wood MJ. Exosomes for targeted siRNA delivery across biological barriers. Adv Drug Deliv Rev. 2013; 65(3):391-397. [PubMed: 22921840]

39. Lee Y, El Andaloussi S, Wood MJ. Exosomes and microvesicles: extracellular vesicles for genetic information transfer and gene therapy. Human molecular genetics. 2012; 21(R1):R125-134. [PubMed: 22872698]

40. Hu CM, Zhang L, Aryal S, Cheung C, Fang RH. Erythrocyte membrane-camouflaged polymeric nanoparticles as a biomimetic delivery platform. Proc Natl Acad Sci U S A. 2011; 108(27):1098010985. [PubMed: 21690347]

41. Jang SC, Kim OY, Yoon CM, et al. Bioinspired Exosome-Mimetic Nanovesicles for Targeted Delivery of Chemotherapeutics to Malignant Tumors. ACS Nano. 2013

42. Lai RC, Arslan F, Lee MM, et al. Exosome secreted by MSC reduces myocardial ischemia/ reperfusion injury. Stem cell research. 2010; 4(3):214-222. [PubMed: 20138817]

43. Haney MJ, Suresh P, Zhao Y, et al. Blood-borne macrophage-neural cell interactions hitchhike endosome networks for cell-based nanozyme brain delivery. Nanomedicine (Lond). 2012; 7(6): 815-833. [PubMed: 22236307]

44. Haney MJ, Zhao Y, Harrison EB, et al. Specific Transfection of Inflamed Brain by Macrophages: A New Therapeutic Strategy for Neurodegenerative Diseases. PloS one. 2013; 8(4):e61852. [PubMed: 23620794]

45. Haney MJ, Zhao Y, Li S, et al. Cell-mediated transfer of catalase nanoparticles from macrophages to brain endothelial, glial and neuronal cells. Nanomedicine (Lond). 2011; 6(7):1215-1230. [PubMed: 21449849]

46. Brynskikh AM, Zhao Y, Mosley RL, et al. Macrophage delivery of therapeutic nanozymes in a murine model of Parkinson's disease. Nanomedicine (Lond). 2010; 5(3):379-396. [PubMed: 20394532] 
47. Brinton RD. A women's health issue: Alzheimer's disease and strategies for maintaining cognitive health. Int J Fertil Womens Med. 1999; 44(4):174-185. [PubMed: 10499738]

48. Gozes I. Neuroprotective peptide drug delivery and development: potential new therapeutics. Trends Neurosci. 2001; 24(12):700-705. [PubMed: 11718874]

49. Kroll RA, Neuwelt EA. Outwitting the blood-brain barrier for therapeutic purposes: osmotic opening and other means. Neurosurgery. 1998; 42(5):1083-1099. discussion 1099-1100. [PubMed: 9588554]

50. Bachis A, Mocchetti I. Brain-Derived Neurotrophic Factor Is Neuroprotective against Human Immunodeficiency Virus-1 Envelope Proteins. Ann N Y Acad Sci. 2005; 1053:247-257. [PubMed: 16179530]

51. Ying Wang J, Peruzzi F, Lassak A, et al. Neuroprotective effects of IGF-I against TNFalphainduced neuronal damage in HIV-associated dementia. Virology. 2003; 305(1):66-76. [PubMed: 12504542]

52. Koliatsos VE, Clatterbuck RE, Nauta HJ, et al. Human nerve growth factor prevents degeneration of basal forebrain cholinergic neurons in primates. Ann Neurol. 1991; 30(6):831-840. [PubMed: 1789695]

53. Dogrukol-Ak D, Banks WA, Tuncel N, Tuncel M. Passage of vasoactive intestinal peptide across the blood-brain barrier. Peptides. 2003; 24(3):437-444. [PubMed: 12732342]

54. Desnick RJ, Schuchman EH. Enzyme replacement and enhancement therapies: lessons from lysosomal disorders. Nat Rev Genet. 2002; 3(12):954-966. [PubMed: 12459725]

55. Urayama A, Grubb JH, Sly WS, Banks WA. Developmentally regulated mannose 6-phosphate receptor-mediated transport of a lysosomal enzyme across the blood-brain barrier. Proc Natl Acad Sci U S A. 2004; 101(34):12658-12663. [PubMed: 15314220]

56. Dou H, Destache CJ, Morehead JR, et al. Development of a macrophage-based nanoparticle platform for antiretroviral drug delivery. Blood. 2006; 108(8):2827-2835. [PubMed: 16809617]

57. Pruszak J, Just L, Isacson O, Nikkhah G. Isolation and culture of ventral mesencephalic precursor cells and dopaminergic neurons from rodent brains. Current protocols in stem cell biology. 2009; 5 Chapter 2, Unit 2D.

58. Bronich T, Nguyen H, Eisenberg A, Kabanov A. Recognition of DNA topology in reactions between plasmid DNA and cationic copolymers. Journal of American Chemical Society. 2000; 122(35):8339-8343.

59. Vinogradov S, Batrakova E, Kabanov A. Poly(ethylene glycol)-polyethyleneimine NanoGel (TM) particles: novel drug delivery systems for antisense oligonucleotides. Colloids and Surfaces BBiointerfaces. 1999; 16(1-4):291-304.

60. Zhao Y, Haney MJ, Klyachko NL, et al. Polyelectrolyte complex optimization for macrophage delivery of redox enzyme nanoparticles. Nanomedicine (Lond). 2011; 6(1):25-42. [PubMed: 21182416]

61. Giovagnoli S, Blasi P, Ricci M, Rossi C. Biodegradable microspheres as carriers for native superoxide dismutase and catalase delivery. AAPS PharmSciTech. 2004; 5(4):e51. [PubMed: 15760048]

62. Batrakova EV, Li S, Reynolds AD, et al. A macrophage-nanozyme delivery system for Parkinson's disease. Bioconjug Chem. 2007; 18(5):1498-1506. [PubMed: 17760417]

63. Tan JW, Tham CL, Israf DA, Lee SH, Kim MK. Neuroprotective effects of biochanin a against glutamate-induced cytotoxicity in PC12 cells via apoptosis inhibition. Neurochemical research. 2013; 38(3):512-518. [PubMed: 23224778]

64. Batrakova EV, Vinogradov SV, Robinson SM, Niehoff ML, Banks WA, Kabanov AV. Polypeptide point modifications with fatty acid and amphiphilic block copolymers for enhanced brain delivery. Bioconjug Chem. 2005; 16(4):793-802. [PubMed: 16029020]

65. Zhao Y, Haney MJ, Mahajan V, et al. Active Targeted Macrophage-mediated Delivery of Catalase to Affected Brain Regions in Models of Parkinson's Disease. J Nanomed Nanotechnol. 2011; S4

66. Tieu K, Perier C, Caspersen C, et al. D-beta-hydroxybutyrate rescues mitochondrial respiration and mitigates features of Parkinson disease. J Clin Invest. 2003; 112(6):892-901. [PubMed: 12975474] 
67. Keshet GI, Tolwani RJ, Trejo A, et al. Increased host neuronal survival and motor function in BMT Parkinsonian mice: involvement of immunosuppression. The Journal of comparative neurology. 2007; 504(6):690-701. [PubMed: 17722033]

68. Papathanou M, Rose S, Mccreary A, Jenner P. Induction and expression of abnormal involuntary movements is related to the duration of dopaminergic stimulation in 6-OHDA-lesioned rats. Eur J Neurosci. 2011; 33(12):2247-2254. [PubMed: 21615558]

69. Papadopoulos S, Jurgens KD, Gros G. Protein diffusion in living skeletal muscle fibers: dependence on protein size, fiber type, and contraction. Biophys J. 2000; 79(4):2084-2094. [PubMed: 11023912]

70. Zhou YZ, Alany RG, Chuang V, Wen J. Optimization of PLGA nanoparticles formulation containing L-DOPA by applying the central composite design. Drug development and industrial pharmacy. 2013; 39(2):321-330. [PubMed: 22607101]

71. Kucherianu VG, Iurasov VV, Kryzhanovskii GN, et al. [The effect of liposomal form of L-Dopa on the development of parkinsonian syndrome in mice]. Biulleten' eksperimental'noi biologii i meditsiny. 1997; 123(1):29-33.

72. Stone DK, Reynolds AD, Mosley RL, Gendelman HE. Innate and Adaptive Immunity for the Pathobiology of Parkinson's Disease. Antioxid Redox Signal. 2009

73. Fruhbeis C, Frohlich D, Kramer-Albers EM. Emerging roles of exosomes in neuron-glia communication. Frontiers in physiology. 2012; 3:119. [PubMed: 22557979]

74. Lotvall J, Valadi H. Cell to cell signalling via exosomes through esRNA. Cell adhesion \& migration. 2007; 1(3):156-158. [PubMed: 19262134]

75. Jin H, Kanthasamy A, Ghosh A, Anantharam V, Kalyanaraman B, Kanthasamy AG. Mitochondriatargeted antioxidants for treatment of Parkinson's disease: preclinical and clinical outcomes. Biochim Biophys Acta. 2014; 1842(8):1282-1294. [PubMed: 24060637]

76. Lucin KM, Wyss-Coray T. Immune activation in brain aging and neurodegeneration: too much or too little? Neuron. 2009; 64(1):110-122. [PubMed: 19840553]

77. Chan PH. Reactive oxygen radicals in signaling and damage in the ischemic brain. J Cereb Blood Flow Metab. 2001; 21(1):2-14. [PubMed: 11149664]

78. Wu DC, Jackson-Lewis V, Vila M, et al. Blockade of microglial activation is neuroprotective in the 1-methyl-4-phenyl-1,2,3,6-tetrahydropyridine mouse model of Parkinson disease. J Neurosci. 2002; 22(5):1763-1771. [PubMed: 11880505]

79. Du Y, Ma Z, Lin S, et al. Minocycline prevents nigrostriatal dopaminergic neurodegeneration in the MPTP model of Parkinson's disease. Proc Natl Acad Sci U S A. 2001; 98(25):14669-14674. [PubMed: 11724929]

80. Kurkowska-Jastrzebska I, Babiuch M, Joniec I, Przybylkowski A, Czlonkowski A, Czlonkowska A. Indomethacin protects against neurodegeneration caused by MPTP intoxication in mice. Int Immunopharmacol. 2002; 2(8):1213-1218. [PubMed: 12349958]

81. Teismann P, Ferger B. Inhibition of the cyclooxygenase isoenzymes COX-1 and COX-2 provide neuroprotection in the MPTP-mouse model of Parkinson's disease. Synapse. 2001; 39(2):167-174. [PubMed: 11180504]

82. Ferger B, Teismann P, Earl CD, Kuschinsky K, Oertel WH. Salicylate protects against MPTPinduced impairments in dopaminergic neurotransmission at the striatal and nigral level in mice. Naunyn Schmiedebergs Arch Pharmacol. 1999; 360(3):256-261. [PubMed: 10543426]

83. Ferger B, Spratt C, Earl CD, Teismann P, Oertel WH, Kuschinsky K. Effects of nicotine on hydroxyl free radical formation in vitro and on MPTP-induced neurotoxicity in vivo. Naunyn Schmiedebergs Arch Pharmacol. 1998; 358(3):351-359. [PubMed: 9774223]

84. Peng J, Stevenson FF, Doctrow SR, Andersen JK. Superoxide dismutase/catalase mimetics are neuroprotective against selective paraquat-mediated dopaminergic neuron death in the substantial nigra: implications for Parkinson disease. J Biol Chem. 2005; 280(32):29194-29198. [PubMed: 15946937]

85. Prasad KN, Cole WC, Hovland AR, et al. Multiple antioxidants in the prevention and treatment of neurodegenerative disease: analysis of biologic rationale. Curr Opin Neurol. 1999; 12(6):761-770. [PubMed: 10676761] 
86. Gonzalez-Polo RA, Soler G, Rodriguezmartin A, Moran JM, Fuentes JM. Protection against MPP+ neurotoxicity in cerebellar granule cells by antioxidants. Cell Biol Int. 2004; 28(5):373-380. [PubMed: 15193280]

87. Pappert EJ, Tangney CC, Goetz CG, et al. Alpha-tocopherol in the ventricular cerebrospinal fluid of Parkinson's disease patients: dose-response study and correlations with plasma levels. Neurology. 1996; 47(4):1037-1042. [PubMed: 8857741]

88. Jamur MC, Oliver C. Permeabilization of cell membranes. Methods Mol Biol. 2010; 588:63-66. [PubMed: 20012820]

89. Rana S, Yue S, Stadel D, Zoller M. Toward tailored exosomes: the exosomal tetraspanin web contributes to target cell selection. The international journal of biochemistry \& cell biology. 2012; 44(9):1574-1584. [PubMed: 22728313]

90. Danhier F, Ansorena E, Silva JM, Coco R, Le Breton A, Preat V. PLGA-based nanoparticles: an overview of biomedical applications. J Control Release. 2012; 161(2):505-522. [PubMed: 22353619]

91. Spuch C, Navarro C. Liposomes for Targeted Delivery of Active Agents against Neurodegenerative Diseases (Alzheimer's Disease and Parkinson's Disease). Journal of drug delivery. 2011; 2011:469679. [PubMed: 22203906]

92. Braak H, Del Tredici K, Rub U, De Vos RA, Jansen Steur EN, Braak E. Staging of brain pathology related to sporadic Parkinson's disease. Neurobiology of aging. 2003; 24(2):197-211. [PubMed: 12498954]

93. Muller FJ, Snyder EY, Loring JF. Gene therapy: can neural stem cells deliver? Nat Rev Neurosci. 2006; 7(1):75-84. [PubMed: 16371952] 


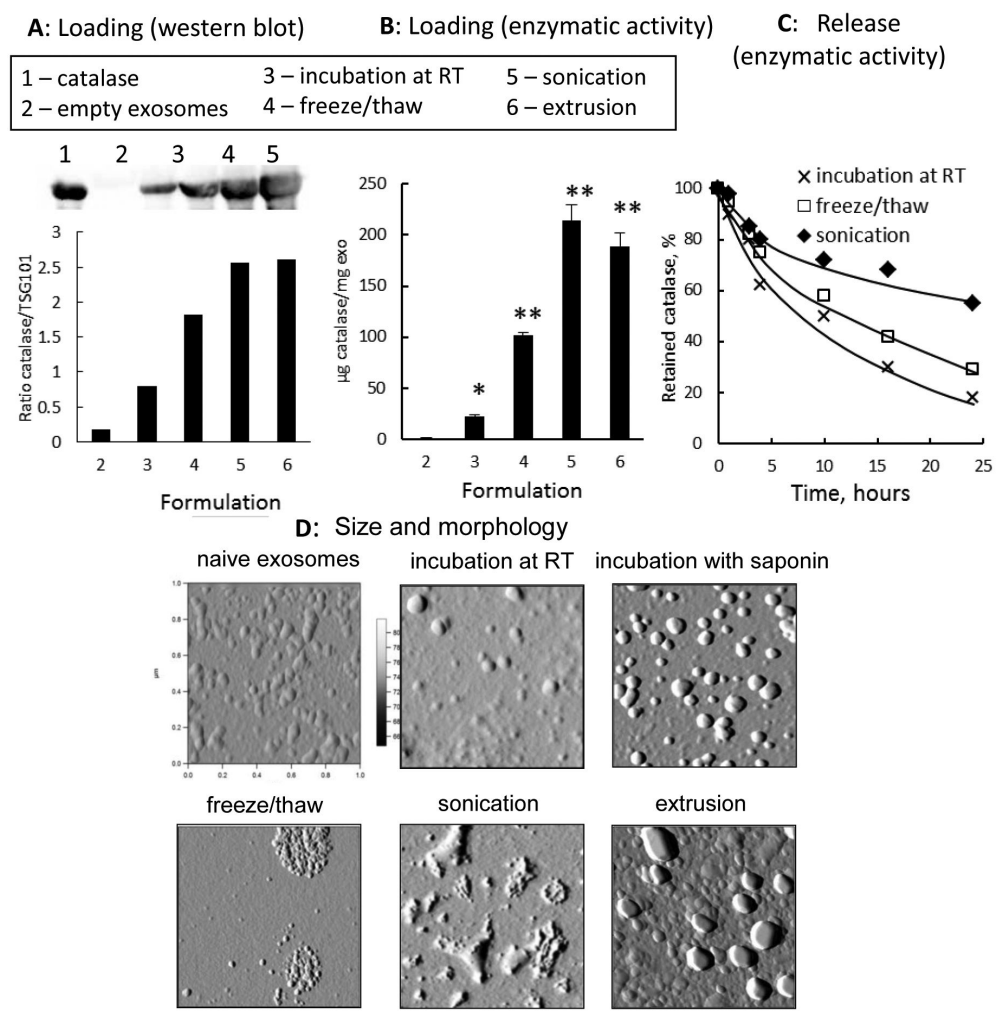

Figure 1. Characterization of exoCAT

Exosomes released from Raw 264.7 macrophages were loaded with catalase by different techniques and examined by: (A) western blot, (B) catalase 10 enzymatic activity; and (C) catalase release. ExoCAT morphology was examined by AFM (D). 


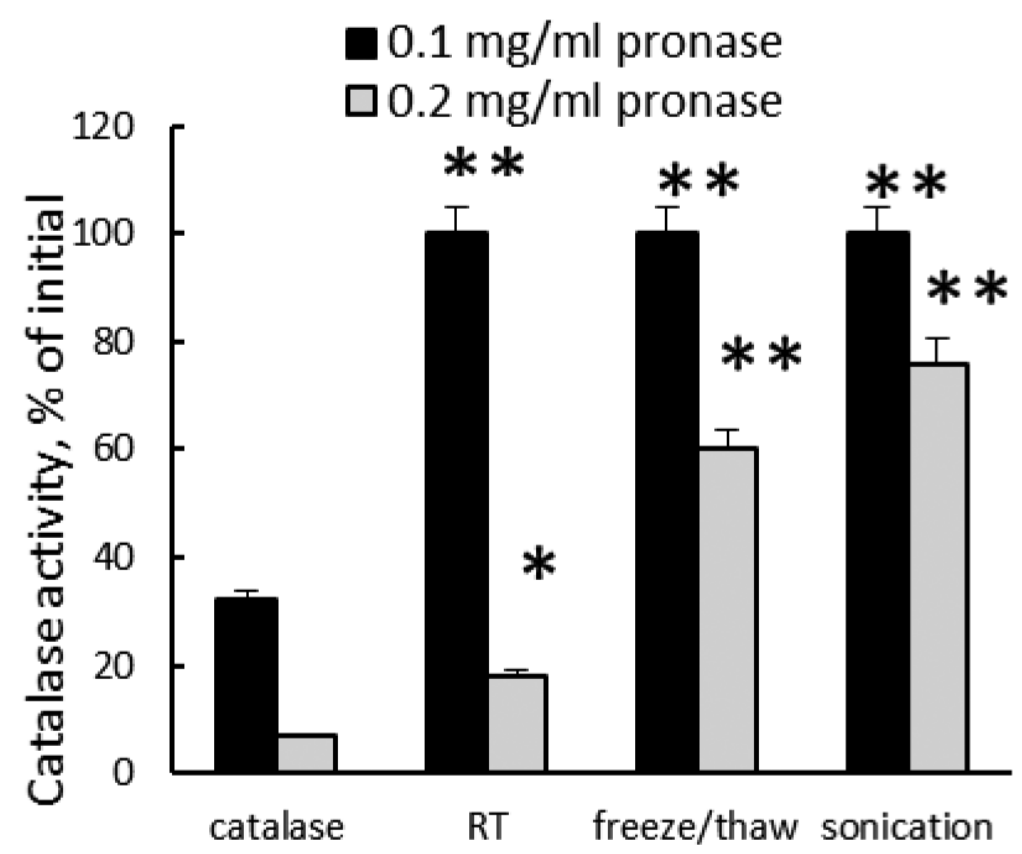

Figure 2. Preservation of catalase enzymatic activity in exoCAT

ExoCAT obtained by sonication demonstrated the best protection of catalase. 

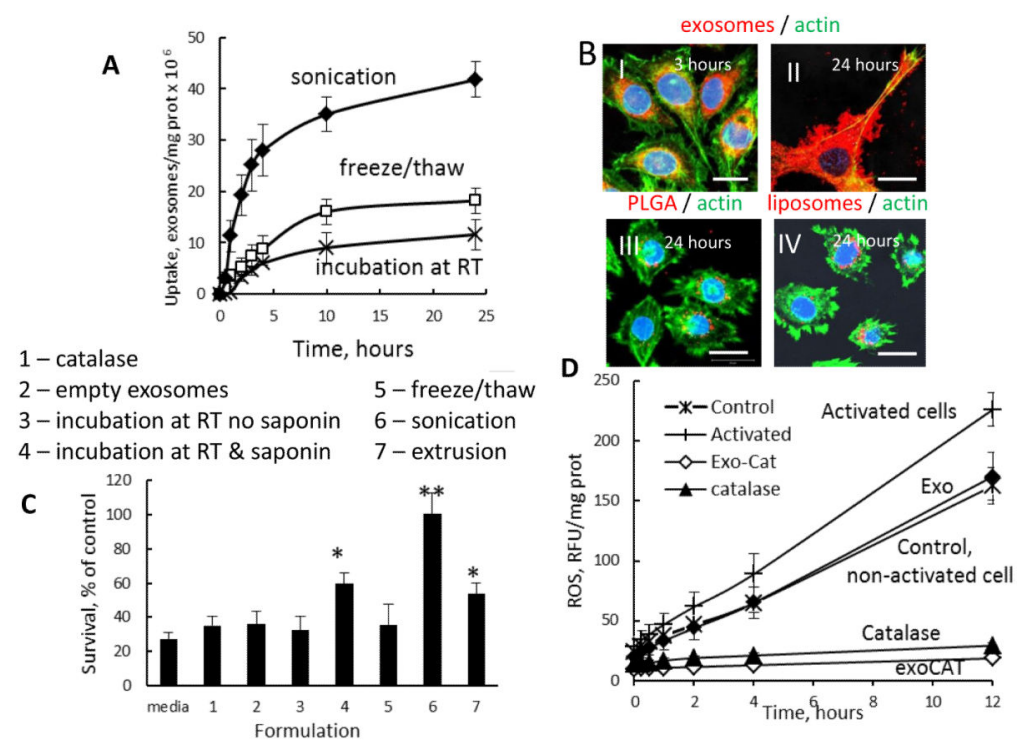

Figure 3. Accumulation of exoCAT in PC12 cells, and therapeutic effects of exoCAT in in vitro models of oxidative stress

The exoCAT uptake in PC12 cells was examined by spectrofluorimetry (A), and confocal microscopy (B). The bar: $20 \mu \mathrm{m}$. The neuroprotection by exoCAT formulations was evaluated in the cell pre-incubated with 6-OHDA (C); (1) catalase, (2) empty exosomes, catalase loaded into exosomes by: (3) incubation at RT, (4) saponin permeabilization, (5) freeze/thaw cycles, (6) sonication, (7) extrusion. The ability to decrease levels of ROS produced in activated macrophages (pre-incubated with LPS and TNF-a) by exoCAT was evaluated by Ampex Red assay in vitro (D). 

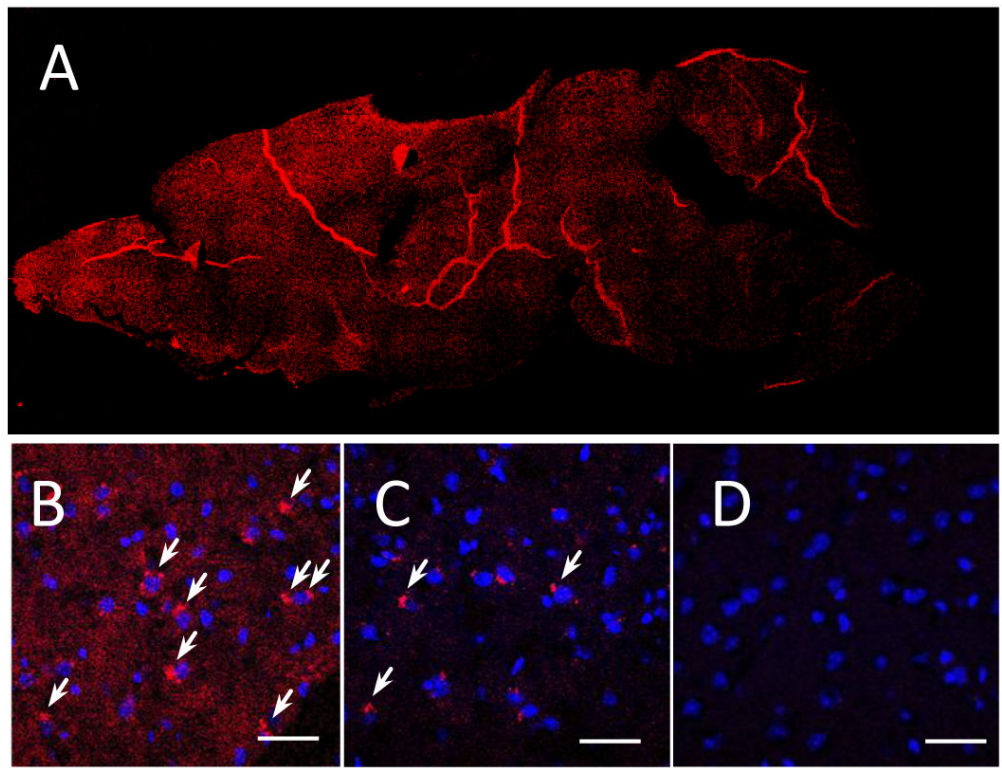

Figure 4. Biodistribution of DIL-labeled exosomes in mouse brain Exosomes were administered to mice with 6-OHDA-induced brain inflammation through: intranasal (A, B), or intravenous $(\mathbf{C})$ routs; and compared to PBS-injected controls $(\mathbf{D})$. The bar: $40 \mu \mathrm{m}$. 

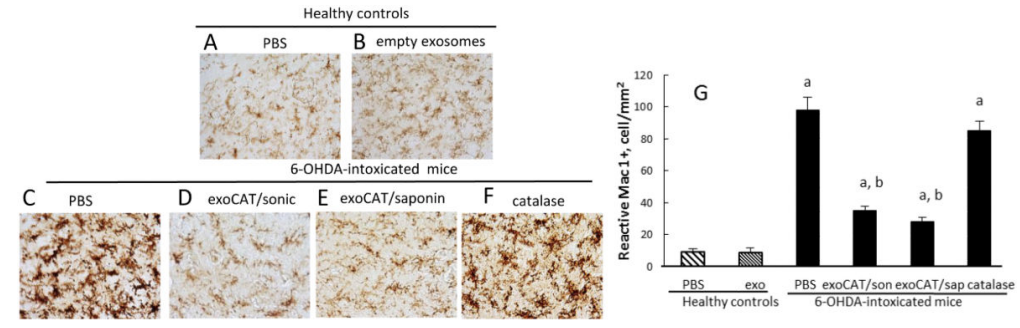

Figure 5. Anti-inflammatory effects of exoCAT in PD mouse model

The intranasal administration of exoCAT significantly decreased microglial activation (D, E) in 6-OHDA-intoxicated mice compared to those intoxicated with 6-OHDA and then treated with PBS $(\mathbf{C})$. Catalase alone did not decrease inflammation in PD mice $(\mathbf{F})$. Empty exosomes did not alter the microglial status in healthy animals (B) compared to healthy controls (A). The anti-inflammatory effects of the described exosomal formulations were quantified by the amount of activated microglial cells $(\mathbf{G})$. 

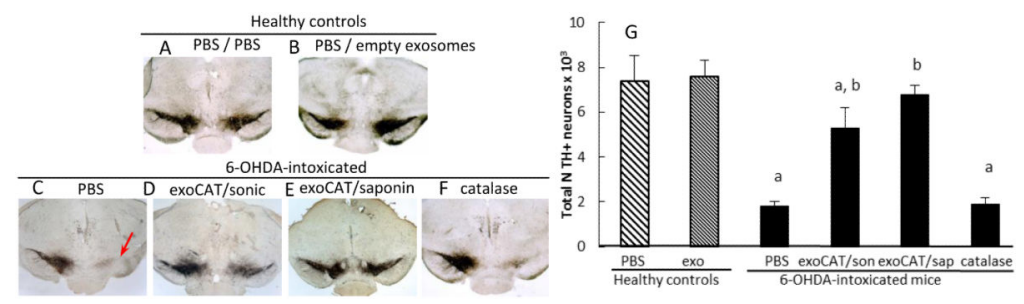

Figure 6. Neuroprotective effects of exoCAT in PD mouse model

The i.n. administration of exoCAT protected DA neurons (D, E) in 6-OHDA-intoxicated mice compared to those intoxicated with 6-OHDA and then treated with PBS $(\mathbf{C})$. Catalase alone was not efficient in this model $(\mathbf{F})$. Empty exosomes did not affect the number of DA neurons in healthy animals (B) compared to healthy controls (A) indicating the absence of cytotoxic effects of the exosomal carriers. The neuroprotective effects of the described exosomal formulations were quantified by the amount of DA neurons in the $\operatorname{SNpc}(\mathbf{G})$. 


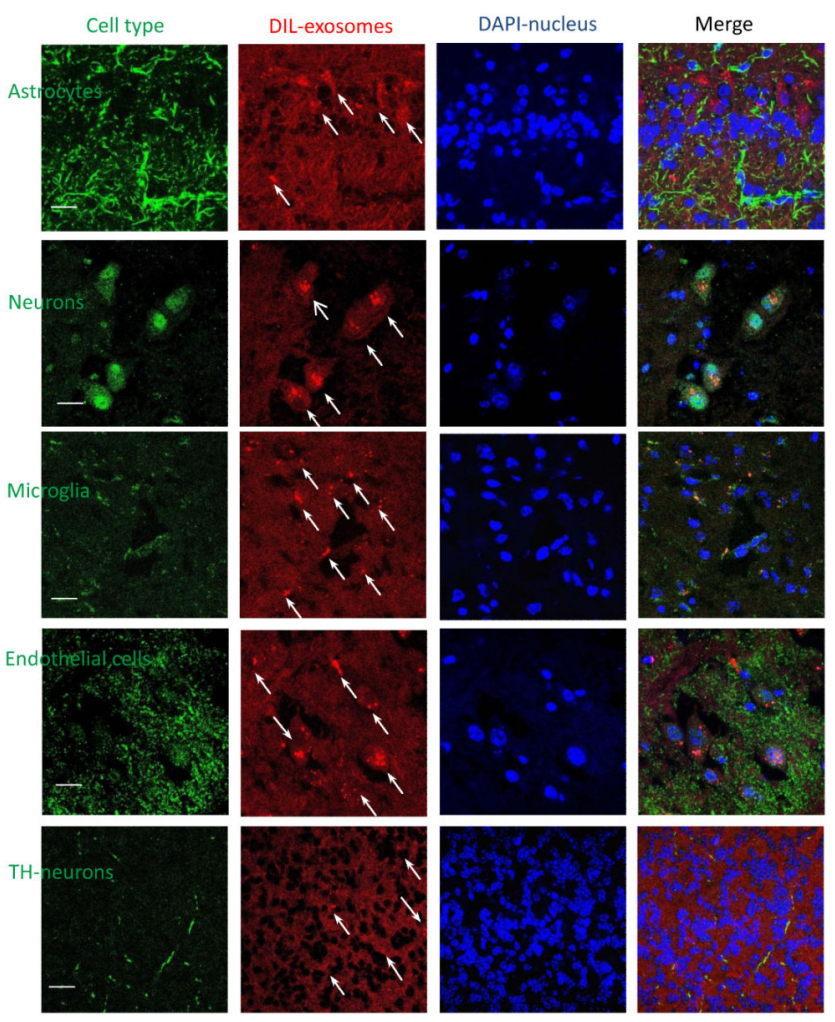

Figure 7. Co-localization of exosomes with different cells in the mouse brain with inflammation Exosomes released by BMM were labeled with DIL (red). C57BL/6 mice were intoxicated with 6-OHDA, and then i.n. injected with fluorescently-labeled exosomes. Four hours later, mice were sacrificed, perfused, and brain slides were subjected for confocal examinations. Brain slides were stained with antibodies to different cell types and then secondary ab 594 (green). Nucleus was stained with DAPI (blue). Bar: $10 \mu \mathrm{m}$. 


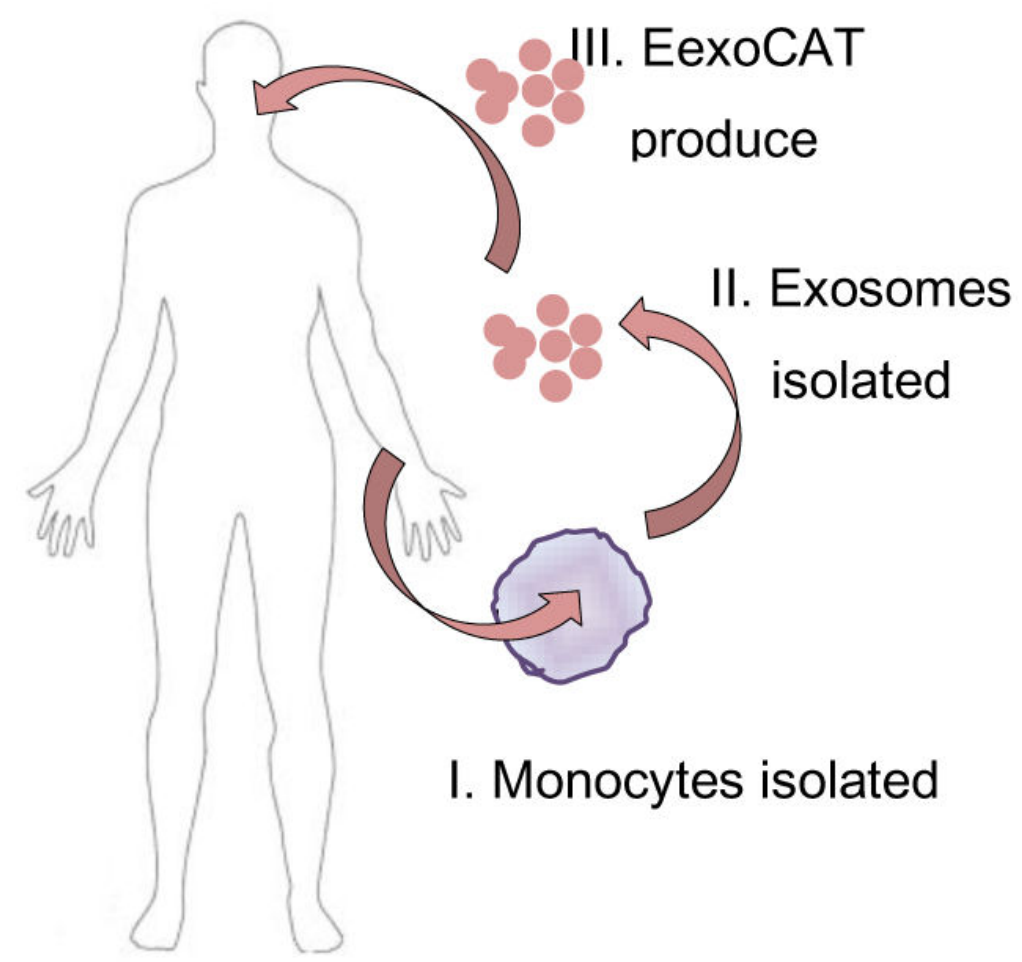

Figure 8. The flow of exoCAT formulations in clinic 
Table 1

Size of catalase exosomal formulations obtained by DLS and NTA ${ }^{a}$

\begin{tabular}{|c|c|c|c|}
\hline \multirow{2}{*}{ Formulation } & \multicolumn{2}{|l|}{ DLS } & \multirow[t]{2}{*}{ NTA (nm) } \\
\hline & $\mathbf{D}_{e f f}(\mathbf{n m})$ & $P d I$ & \\
\hline Catalase alone & $9.5 \pm 0.1$ & 0.10 & $\mathrm{n} / \mathrm{a}$ \\
\hline exosomes alone & $100.5 \pm 13.5$ & 0.20 & $99.5 \pm 11.2$ \\
\hline exoCAT, mixture & $108.0 \pm 14.3(\mathrm{~ns})$ & 0.35 & $100 \pm 16.3(\mathrm{~ns})$ \\
\hline exoCAT, mixture \& saponin & $110.5 \pm 23.1(\mathrm{~ns})$ & 0.35 & $111 \pm 7.8(\mathrm{~ns})$ \\
\hline exosomes, freeze/thaw & $147.0 \pm 10.0^{(*)}$ & 0.48 & $125 \pm 17^{(*)}$ \\
\hline exoCAT, freeze/thaw & $158.0 \pm 11.0^{(*)}$ & 0.48 & $130 \pm 11^{(*)}$ \\
\hline exosomes, sonicated & $179.0 \pm 10.6^{(*)}$ & 0.30 & $150 \pm 8.2^{(*)}$ \\
\hline exoCAT, sonicated & $183.7 \pm 13.8^{(* *)}$ & 0.25 & $162.4 \pm 6.1^{(* *)}$ \\
\hline exosomes, extruded & $134.0 \pm 7.5^{(*)}$ & 0.25 & $130 \pm 7.5^{(*)}$ \\
\hline exoCAT, extruded & $154.8 \pm 11.0^{(*)}$ & 0.29 & $149.4 \pm 3.3^{(*)}$ \\
\hline
\end{tabular}

${ }^{a}$ Statistical significance is compared to the diameter of naïve exosomes alone and shown by symbols:

${ }^{*}{ }_{p}<0.05$

${ }^{* *}{ }_{p}<0.005$ 